\title{
Dehydroalkylative Activation of CNN- and PNN-Pincer Ruthenium Catalysts for Ester Hydrogenation
}

Tianyi He, John C. Buttner, Eamon F. Reynolds, John Pham, Jack C. Malek, Jason M. Keith,* and Anthony R. Chianese*

\section{Table of Contents}

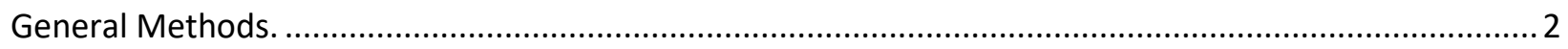

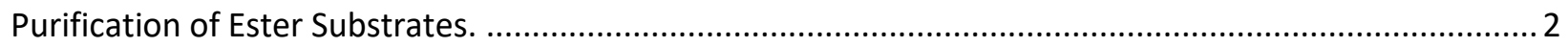

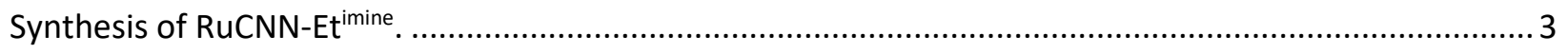

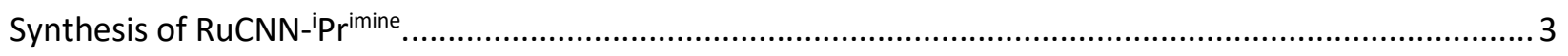

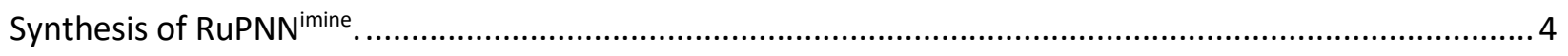

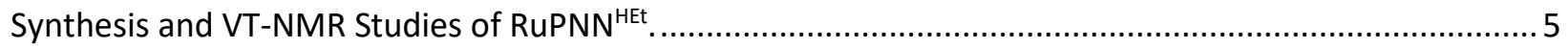

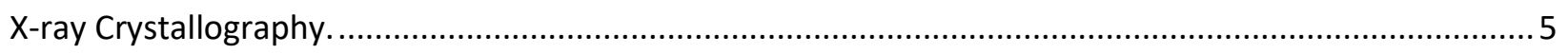

Apparatus for Time-Course Monitoring of Hydrogenation Reactions.................................................... 5

Experimental Procedure for Time-Course Monitoring of Hydrogenation Reactions. ............................... 6

Measurement of Ethane and Propane Formation During Hydrogenation Reactions. .............................. 6

Hydrogenation Substrate Scope Experiments in a Stainless-Steel Pressure Reactor................................ 7

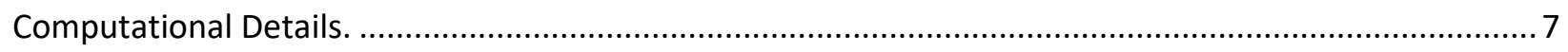

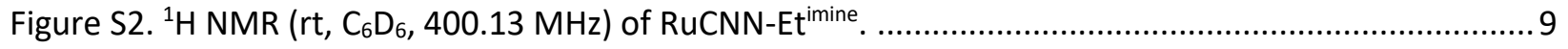

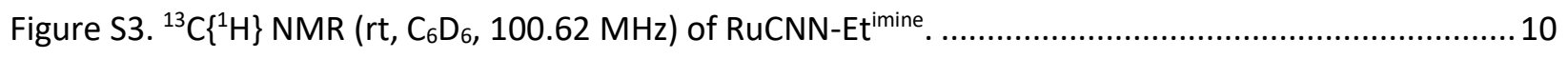

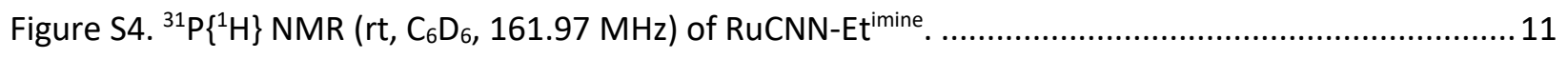

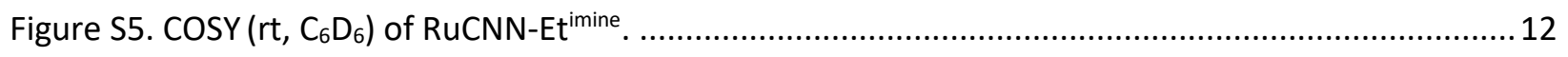

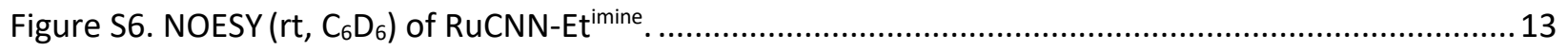

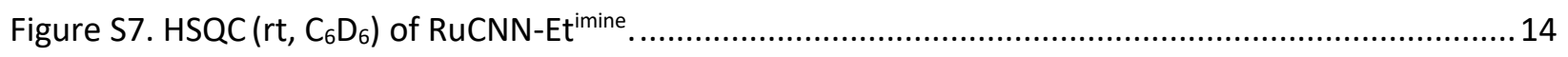

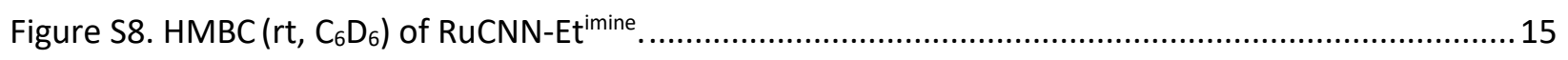

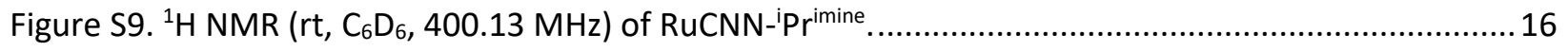

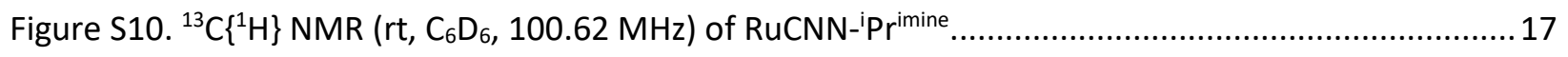

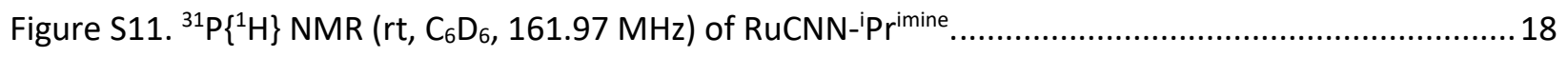




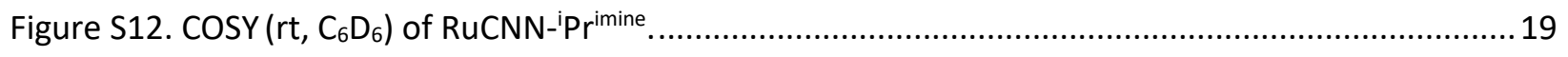

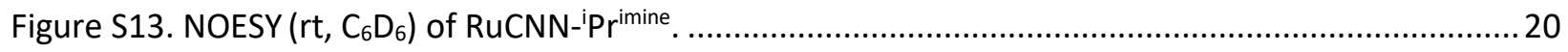

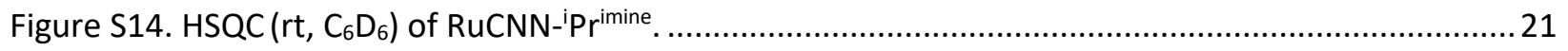

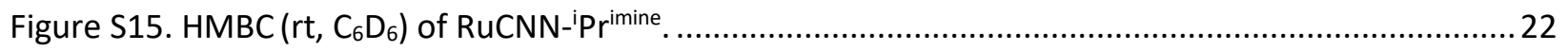

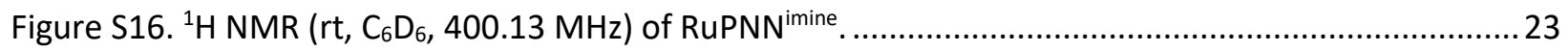

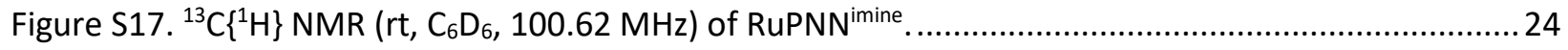

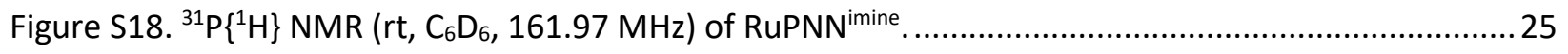

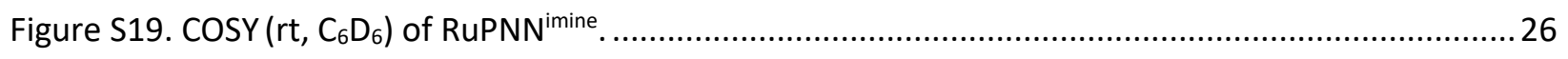

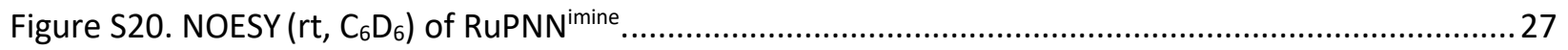

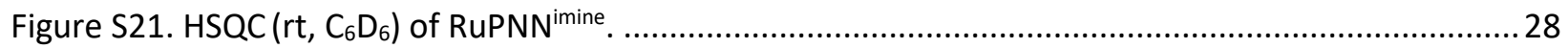

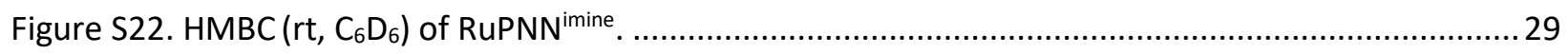

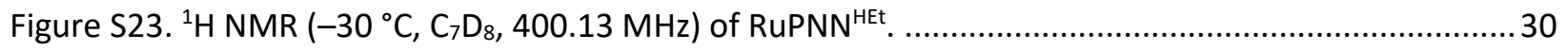

Figure S24. ${ }^{1} \mathrm{H}\left\{{ }^{31} \mathrm{P}\right\}$ NMR $\left(-30{ }^{\circ} \mathrm{C}, \mathrm{C}_{7} \mathrm{D}_{8}, 400.13 \mathrm{MHz}\right)$ of RuPNN ${ }^{\mathrm{HEt}}$ (top), compared with the undecoupled

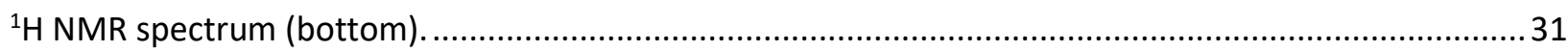

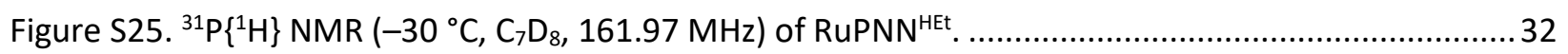

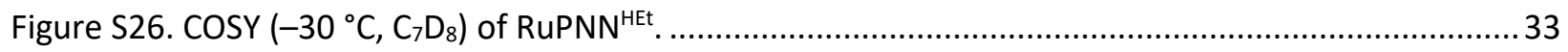

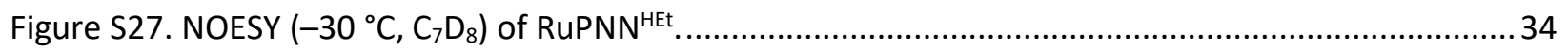

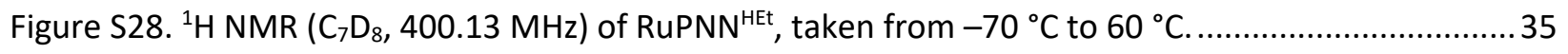

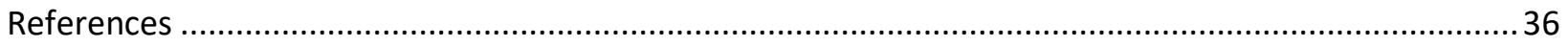

General Methods. Unless stated otherwise, all reactions were assembled in an argon-filled MBraun Labmaster 130 glovebox. Solvents were purchased in anhydrous form from EMD-Millipore or Acros and were deoxygenated by sparging with argon before bringing into the glovebox. All reagents and materials were commercially available and were used as received, unless otherwise noted. RuPNN ${ }^{\text {dearom }}$ and RuPNN $^{\text {HCl }}$ were obtained from Strem. Hydrogen gas was purchased from Airgas at the Ultrahigh Purity level. NMR spectra were recorded at room temperature (unless otherwise noted) on a Bruker spectrometer (400.13 MHz for ${ }^{1} \mathrm{H}$ and $100.62 \mathrm{MHz}$ for ${ }^{13} \mathrm{C}, 161.97 \mathrm{MHz}$ for ${ }^{31} \mathrm{P}$ ) and referenced to the residual solvent resonance ( $\delta$ in parts per million, $\mathrm{J}$ in $\mathrm{Hz}$ ). NMR assignments were made on the basis of COSY, NOESY, HSQC, and HMBC spectra, which are included as images in this document. Gas chromatography was conducted using a Shimadzu 2030 system equipped with an FID detector. Elemental analyses were performed by Robertson Microlit, Madison, NJ.

Purification of Ester Substrates. When commercial samples of ester substrates were employed in basefree catalytic hydrogenation, we found that removal of dissolved oxygen by sparging was not sufficient to obtain optimal catalytic activity, most likely due to small amounts of carboxylic acid impurities in the 
substrates. To obtain optimal yields at low catalyst loadings and reproducible rates in kinetic experiments, substrates were purified by chromatography prior to use. For ethyl benzoate, ethyl cyclohexanecarboxylate, methyl cyclohexanecarboxylate, methyl 3-cyclohexenecarboxylate, benzyl benzoate, methyl decanoate, methyl benzoate, hexyl hexanoate, phthalide, and methyl cinnamate, commcerically purchased substrates were purified by flash chromatography on silica gel, eluting with a gradient of 0-20\% MTBE in hexanes. Flash chromatography was conducted using a Combiflash RF system, and esters were detected for fraction collection by their UV absorbance at $205 \mathrm{~nm}$. (S)-Ethyl ibuprofen was synthesized as previously described, ${ }^{1}$ but was additionally purified using the same chromatographic procedure. Gusev has previously reported the purification of ester substrates by filtration through activated basic alumina. ${ }^{2}$ We found this method to be successful as well and employed it for methyl 10-undecenoate. After chromatographic purification, esters were degassed (sparging with argon for liquids or prolonged evacuation for solids) before bringing into the glovebox.

Synthesis of RuCNN-Et ${ }^{\text {imine }}$. In the glovebox, RuCNN-Et ${ }^{\mathrm{HCl} 3}$ (160 mg, $0.26 \mathrm{mmol}$ ), tricyclohexylphosphine (77 mg, $0.28 \mathrm{mmol}$ ), and sodium $t$-butoxide $(51 \mathrm{mg}, 0.53 \mathrm{mmol}$ ) were added to a $10 \mathrm{~mL}$ vial along with a stirbar. Mesitylene $(2.5 \mathrm{~mL})$ was added, and the mixture was heated to $140{ }^{\circ} \mathrm{C}$ for two hours. The mixture was allowed to cool, and was filtered through a PTFE filter disk into a $20 \mathrm{~mL}$ vial, washing through with $1 \mathrm{~mL}$ toluene. The solution was layered with $15 \mathrm{~mL}$ acetonitrile, causing the slow formation of deep green crystals. After two days, the supernatant was carefully decanted away, and the remaining solid was washed 10 times with a 4:1 mixture of acetonitrile and toluene. The dark green, highly airsensitive solid product was dried under vacuum and stored in the glovebox at room temperature. Yield: $123 \mathrm{mg}, 57 \% .{ }^{1} \mathrm{H}$ NMR $\left(\mathrm{C}_{6} \mathrm{D}_{6}, 400.13 \mathrm{MHz}, 22{ }^{\circ} \mathrm{C}\right): \delta 8.08\left(\mathrm{~d},{ }^{4} \mathrm{~J}_{\mathrm{HP}}=5.0 \mathrm{~Hz}, 1 \mathrm{H}, \mathrm{CH}_{\text {imine }}\right) ; 7.83\left(\mathrm{~d},{ }^{3} \mathrm{~J}_{\mathrm{HH}}=8.0\right.$ $\left.\mathrm{Hz}, 1 \mathrm{H}, \mathrm{CH}_{\text {imid }}\right) ; 7.61\left(\mathrm{~d},{ }^{3} \mathrm{~J}_{\mathrm{HH}}=8.2 \mathrm{HZ}, 1 \mathrm{H}, \mathrm{CH}_{\text {pyr }}\right) ; 7.45-7.39\left(\mathrm{~m}, 3 \mathrm{H}, \mathrm{CH}_{\text {pyr }}\right.$ and $\left.\mathrm{CH}_{\text {dipp }}\right) ; 7.26\left(\mathrm{~m}, 1 \mathrm{H}, \mathrm{CH}_{\text {dipp }}\right)$; $7.18\left(\mathrm{~m}, 1 \mathrm{H}, \mathrm{CH}_{\text {pyr }}\right) ; 7.02\left(\mathrm{t},{ }^{3} J_{\mathrm{HH}}=8.0 \mathrm{~Hz}, 1 \mathrm{H}, \mathrm{CH}_{\text {imid }}\right) ; 6.89\left(\mathrm{t},{ }^{3} J_{\mathrm{HH}}=8.0 \mathrm{~Hz}, 1 \mathrm{H}, \mathrm{CH}_{\text {imid }}\right) ; 6.55\left(\mathrm{~d},{ }^{3} J_{\mathrm{HH}}=8.0\right.$ $\mathrm{Hz}, 1 \mathrm{H}, \mathrm{CH}_{\text {imid }}$ ); 4.79 (dqd, $\left.{ }^{2} J_{\mathrm{HH}}=10.5 \mathrm{~Hz},{ }^{3} J_{\mathrm{HH}}=7.3 \mathrm{~Hz},{ }^{4} J_{\mathrm{HP}}=3.3 \mathrm{~Hz}, 1 \mathrm{H}, \mathrm{CH}_{2-\mathrm{Et}}\right) ; 4.13$ (dqd, ${ }^{2} J_{\mathrm{HH}}=10.5 \mathrm{~Hz}$, ${ }^{3} J_{\mathrm{HH}}=7.1 \mathrm{~Hz},{ }^{4} J_{\mathrm{HP}}=3.2 \mathrm{~Hz}, 1 \mathrm{H}, \mathrm{CH}_{2-\mathrm{Et}}$ ); 3.46 (sept, ${ }^{3} J_{\mathrm{HH}}=6.8 \mathrm{~Hz}, 1 \mathrm{H}, \mathrm{CH}_{\mathrm{iPr}}$ ); 2.14 (sept, ${ }^{3} \mathrm{~J}_{\mathrm{HH}}=6.8 \mathrm{~Hz}, 1 \mathrm{H}$, $\left.\mathrm{CH}_{\mathrm{iPr}}\right) ; 1.59\left(\mathrm{t},{ }^{3} \mathrm{H}_{\mathrm{HH}}=7.2 \mathrm{~Hz}, 3 \mathrm{H}, \mathrm{CH}_{3-\mathrm{Et}}\right) ; 1.58\left(\mathrm{~d},{ }^{3} J_{\mathrm{HH}}=6.8 \mathrm{~Hz}, 3 \mathrm{H}, \mathrm{CH}_{3-\mathrm{Pr}}\right) ; 1.60-1.30(\mathrm{br} \mathrm{m}, \mathrm{PCy})$ ); $1.31(\mathrm{~d}$, $\left.{ }^{3} J_{\mathrm{HH}}=6.8 \mathrm{~Hz}, 3 \mathrm{H}, \mathrm{CH}_{3-\mathrm{Pr}}\right) ; 1.20-0.90(\mathrm{br} \mathrm{m}, \mathrm{PCY} 3) ; 0.95$ (d, $\left.{ }^{3} \mathrm{~J}_{\mathrm{HH}}=6.8 \mathrm{~Hz}, 3 \mathrm{H}, \mathrm{CH}_{3-\mathrm{Pr}}\right) ; 0.49\left(\mathrm{~d},{ }^{3} J_{\mathrm{HH}}=6.8 \mathrm{~Hz}\right.$, $\left.3 \mathrm{H}, \mathrm{CH}_{3-\mathrm{Pr}}\right) .{ }^{13} \mathrm{C}\left\{{ }^{1} \mathrm{H}\right\} \operatorname{NMR}\left(\mathrm{C}_{6} \mathrm{D}_{6}, 100.62 \mathrm{MHz}, 22{ }^{\circ} \mathrm{C}\right): \delta 214.4\left(\mathrm{~d},{ }^{2} \mathrm{~J}_{\mathrm{CP}}=8.3 \mathrm{~Hz}, \mathrm{CO}\right) ; 213.6\left(\mathrm{~d},{ }^{2} \mathrm{~J}_{\mathrm{CP}}=1.6 \mathrm{~Hz}\right.$, $\left.\mathrm{C}_{\mathrm{NHC}}\right) ; 149.8\left(\mathrm{C}_{\text {dipp }}\right) ; 147.1\left(\mathrm{C}_{\text {dipp }}\right) ; 146.4\left(\mathrm{~d},{ }^{3} J_{\mathrm{HH}}=3.0 \mathrm{~Hz}, \mathrm{C}_{\text {pyr }}\right) ; 141.2\left(\mathrm{C}_{\text {pyr }}\right) ; 140.2\left(\mathrm{C}_{\text {imid }}\right) ; 140.0\left(\mathrm{C}_{\text {imine }}\right) ; 135.8$ $\left(C_{\text {dipp }}\right) ; 132.9\left(C_{\text {imid }}\right) ; 130.2\left(C_{\text {dipp }}\right) ; 126.3\left(C_{\text {dipp }}\right) ; 123.8\left(C_{\text {dipp }}\right) ; 122.2\left(C_{\text {imid }}\right) ; 121.1\left(C_{\text {dipp }}\right) ; 118.7\left(d,{ }^{5} J_{C P}=3.0\right.$ $\left.\mathrm{Hz}, \mathrm{C}_{\text {pyr }}\right) ; 116.0\left(\mathrm{~d},{ }^{4} \mathrm{~J}_{\mathrm{CP}}=3.4 \mathrm{~Hz}, \mathrm{C}_{\text {pyr }}\right) ; 110.6\left(\mathrm{C}_{\text {imid }}\right) ; 109.8$ ( $\left.\mathrm{C}_{\text {imid }}\right) ; 94.2\left(\mathrm{C}_{\mathrm{pyr}}\right) ; 60.8\left(\mathrm{~d},{ }^{3} \mathrm{~J}_{\mathrm{CP}}=3.3 \mathrm{~Hz}, \mathrm{C}_{\mathrm{Et}}\right) ; 37.6$ $\left(d,{ }^{1} J_{C P}=13.5 \mathrm{~Hz}, P C y_{3}\right) ; 29.6\left(\mathrm{PCy}_{3}\right) ; 29.0\left(\mathrm{PCy}_{3}\right) ; 28.69\left(\mathrm{CH}_{\mathrm{iPr}}\right) ; 28.65\left(\mathrm{CH}_{\mathrm{iPr}}\right) ; 28.1\left(\mathrm{~d},{ }^{2} \mathrm{JPP}_{\mathrm{CP}}=9.1 \mathrm{~Hz}, \mathrm{PCY}\right)$; $27.9\left(\mathrm{~d},{ }^{2} J_{\mathrm{CP}}=10.6 \mathrm{~Hz}, \mathrm{PCy}\right) ; 26.8\left(\mathrm{CH}_{3-\mathrm{Pr} r}\right) ; 25.0\left(\mathrm{CH}_{3-\mathrm{Pr}}\right) ; 24.9\left(\mathrm{CH}_{3-\mathrm{Pr} r}\right) ; 23.0\left(\mathrm{CH}_{3-\mathrm{Pr} r}\right) ; 22.2\left(\mathrm{~d},{ }^{4} \mathrm{~J}_{\mathrm{CP}}=4.6 \mathrm{~Hz}\right.$, $\left.\mathrm{CH}_{3-\mathrm{Et}}\right) .{ }^{31} \mathrm{P}\left\{{ }^{1} \mathrm{H}\right\} \mathrm{NMR}\left(\mathrm{C}_{6} \mathrm{D}_{6}, 101.97 \mathrm{MHz}, 22{ }^{\circ} \mathrm{C}\right): \delta$ 50.95. Anal calcd. For $\mathrm{C}_{46} \mathrm{H}_{63} \mathrm{~N}_{4} \mathrm{OPRu}: \mathrm{C}, 67.37 ; \mathrm{H}, 7.74$; $\mathrm{N}, 6.83$. Found: $\mathrm{C}, 67.48 ; \mathrm{H}, 7.86 ; \mathrm{N}, 6.92$.

Synthesis of RuCNN-'Primine . In the glovebox, RuCNN-i Pr ${ }^{\mathrm{HCl} 3}$ (340 mg, $0.54 \mathrm{mmol}$ ), tricyclohexylphosphine (158 $\mathrm{mg}, 0.56 \mathrm{mmol}$ ), and sodium $t$-butoxide (103 mg, $1.07 \mathrm{mmol}$ ) were added to a $10 \mathrm{~mL}$ vial along with a stirbar. Mesitylene $(5 \mathrm{~mL})$ was added, and the mixture was heated to $120^{\circ} \mathrm{C}$ overnight. The mixture was allowed to cool, and was filtered through a PTFE filter disk into a $40 \mathrm{~mL}$ vial, washing through with 2 
$\mathrm{mL}$ toluene. The solution was layered with $30 \mathrm{~mL}$ acetonitrile, causing the slow formation of deep green crystals. After two days, the supernatant was carefully decanted away, and the remaining solid was washed 10 times with a 4:1 mixture of acetonitrile and toluene. The dark green, highly air-sensitive solid product was dried under vacuum and stored in the glovebox at room temperature. Yield: $201 \mathrm{mg}, 42 \%$. Even upon prolonged storage under vacuum, crystals of RuCNN-'Primine retained fractional amounts of the solvents present in the recrystallization. The quantities of solvents present in the sample submitted for analysis were analyzed by ${ }^{1} \mathrm{H} N \mathrm{NR}$, and the yield given above is corrected for the detected $6 \%$ solvent impurity by mass. ${ }^{1} \mathrm{H} N M R\left(\mathrm{C}_{6} \mathrm{D}_{6}, 400.13 \mathrm{MHz}, 22{ }^{\circ} \mathrm{C}\right): \delta 8.35\left(\mathrm{~d},{ }^{4} \mathrm{~J}_{\mathrm{HP}}=4.7 \mathrm{~Hz}, 1 \mathrm{H}, \mathrm{CH}_{\text {imine }}\right) ; 7.84(\mathrm{~d}$, $\left.{ }^{3} J_{\mathrm{HH}}=7.8 \mathrm{~Hz}, 1 \mathrm{H}, \mathrm{CH}_{\text {imid }}\right) ; 7.63\left(\mathrm{~d},{ }^{3} \mathrm{~J}_{\mathrm{HH}}=8.2 \mathrm{~Hz}, 1 \mathrm{H}, \mathrm{CH}_{\text {pyr }}\right) ; 7.45-7.38\left(\mathrm{~m}, 3 \mathrm{H}, \mathrm{CH}_{\text {dipp }}\right.$ and $\left.\mathrm{CH}_{\text {pyr }}\right) ; 7.27(\mathrm{~m}, 1 \mathrm{H}$, $\left.\mathrm{CH}_{\text {dipp }}\right) ; 7.19\left(\mathrm{~m}, 1 \mathrm{H}, \mathrm{CH}_{\text {pyr }}\right) ; 7.02\left(\mathrm{t},{ }^{3} \mathrm{~J}_{\mathrm{HH}}=7.8 \mathrm{~Hz}, 1 \mathrm{H}, \mathrm{CH}_{\text {imid }}\right) ; 6.89\left(\mathrm{t},{ }^{3} J_{\mathrm{HH}}=7.8 \mathrm{~Hz}, 1 \mathrm{H}, \mathrm{CH}_{\text {imid }}\right) ; 6.55\left(\mathrm{~d},{ }^{3} J_{\mathrm{HH}}\right.$ $=7.8 \mathrm{~Hz}, 1 \mathrm{H}, \mathrm{CH}_{\text {imid }}$ ); 5.31 (sept, ${ }^{3} \mathrm{JHH}_{\mathrm{HH}}=6.5 \mathrm{~Hz}, 1 \mathrm{H}, \mathrm{CH}_{\mathrm{iPr}}$ ); 3.46 (sept, ${ }^{3} \mathrm{~J}_{\mathrm{HH}}=6.8 \mathrm{~Hz}, 1 \mathrm{H}, \mathrm{CH}_{\mathrm{iPr}}$ ); 2.15 (sept, $\left.{ }^{3} J_{\mathrm{HH}}=6.8 \mathrm{~Hz}, 1 \mathrm{H}, \mathrm{CH}_{\mathrm{iPr}}\right) ; 1.65\left(\mathrm{~d},{ }^{3} \mathrm{JHH}_{\mathrm{HH}}=6.5 \mathrm{~Hz}, 1 \mathrm{H}, \mathrm{CH}_{3-\mathrm{Pr} r}\right) ; 1.60\left(\mathrm{~d}, 3^{3} \mathrm{HH}_{\mathrm{HH}}=6.8 \mathrm{~Hz}, 1 \mathrm{H}, \mathrm{CH}_{\mathrm{iPr}}\right) ; 1.56\left(\mathrm{~d},{ }^{3} J_{\mathrm{HH}}=\right.$ $6.5 \mathrm{~Hz}, 1 \mathrm{H}, \mathrm{CH}_{\mathrm{iPr}}$ ); $1.60-1.25$ (br m, PCy $)$; 1.31 (d, ${ }^{3} \mathrm{~J}_{\mathrm{HH}}=6.8 \mathrm{~Hz}, 1 \mathrm{H}, \mathrm{CH}_{\mathrm{iPr}}$ ); 1.22-0.80 (br m, PCy $)$; 0.95 (d, $\left.{ }^{3} J_{\mathrm{HH}}=6.8 \mathrm{~Hz}, 1 \mathrm{H}, \mathrm{CH}_{\mathrm{iPr}}\right) ; 0.48\left(\mathrm{~d},{ }^{3} \mathrm{~J}_{\mathrm{HH}}=6.8 \mathrm{~Hz}, 1 \mathrm{H}, \mathrm{CH}_{\mathrm{iPr}}\right) .{ }^{13} \mathrm{C}\left\{{ }^{1} \mathrm{H}\right\} \mathrm{NMR}\left(\mathrm{C}_{6} \mathrm{D}_{6}, 100.62 \mathrm{MHz}, 22^{\circ} \mathrm{C}\right): \delta 214.4$ $\left(\mathrm{d},{ }^{2} J_{\mathrm{CP}}=8.0 \mathrm{~Hz}, \mathrm{CO}\right) ; 213.5\left(\mathrm{~d},{ }^{2} J_{\mathrm{CP}}=1.6 \mathrm{~Hz}, \mathrm{C}_{\mathrm{NHC}}\right) ; 149.8\left(\mathrm{C}_{\mathrm{dipp}}\right) ; 147.0\left(\mathrm{C}_{\mathrm{dipp}}\right) ; 146.3\left(\mathrm{~d},{ }^{3} J_{\mathrm{HH}}=3.0 \mathrm{~Hz}, \mathrm{C}_{\mathrm{pyr}}\right)$; 141.8 ( $\left.C_{\text {pyr }}\right) ; 140.2$ ( $\left.C_{\text {imid }}\right) ; 135.8$ ( $\left.C_{\text {dipp }}\right) ; 132.9\left(C_{\text {imid }}\right) ; 130.5$ ( $\left.C_{\text {imine }}\right) ; 130.2$ ( $\left.C_{\text {dipp }}\right) ; 126.4\left(C_{\text {dipp }}\right) ; 123.8$ ( $\left.C_{\text {dipp }}\right)$; 122.2 ( $\left.C_{\text {imid }}\right) ; 121.2$ ( $\left.C_{\text {dipp }}\right) ; 118.5$ (d, $\left.{ }^{5} J_{C P}=3.2 \mathrm{~Hz}, C_{\text {pyr }}\right) ; 116.3$ (d, $\left.{ }^{4} J_{C P}=3.5 \mathrm{~Hz}, C_{\text {pyr }}\right) ; 110.6$ ( $C_{\text {imid }}$ ); 109.7 $\left(C_{\text {imid }}\right) ; 94.2\left(C_{\text {pyr }}\right) ; 64.4\left(d,{ }^{3} J_{C P}=3.1 \mathrm{~Hz}, \mathrm{CH}_{\text {ipr }}\right) ; 37.6\left(\mathrm{br}, \mathrm{PCy}_{3}\right) ; 30.2\left(\mathrm{~d},{ }^{4} \mathrm{~J}_{\mathrm{CP}}=4.9 \mathrm{~Hz}, \mathrm{CH}_{3-\mathrm{iPr}}\right) ; 29.4\left(\mathrm{PCY}_{3}\right)$; $29.0\left(\mathrm{PCY}_{3}\right) ; 28.7\left(\mathrm{CH}_{\mathrm{iPr}}\right) ; 28.6\left(\mathrm{CH}_{\mathrm{iPr}}\right) ; 28.1\left(\mathrm{~d},{ }^{2} \mathrm{~J}_{\mathrm{CP}}=10.0 \mathrm{~Hz}, \mathrm{PCy}\right) ; 26.7\left(\mathrm{PCy}_{3}\right) ; 26.0\left(\mathrm{CH}_{3-\mathrm{Pr}}\right) ; 25.0\left(\mathrm{CH}_{3-\mathrm{Pr} r}\right)$; $24.9\left(\mathrm{CH}_{3-\mathrm{Pr} r}\right) ; 22.9\left(\mathrm{CH}_{3-\mathrm{Pr}}\right) ; 22.5\left(\mathrm{~d},{ }^{4} \mathrm{~J}_{\mathrm{CP}}=1.2 \mathrm{~Hz}, \mathrm{CH}_{3-\mathrm{Pr}}\right) .{ }^{31} \mathrm{P}\left\{{ }^{1} \mathrm{H}\right\} \mathrm{NMR}\left(\mathrm{C}_{6} \mathrm{D}_{6}, 101.97 \mathrm{MHz}, 22{ }^{\circ} \mathrm{C}\right): \delta 52.69$. Anal calcd. For $\mathrm{C}_{46} \mathrm{H}_{63} \mathrm{~N}_{4} \mathrm{OPRu} \cdot\left(0.49 \mathrm{C}_{7} \mathrm{H}_{8}\right) \cdot\left(0.17 \mathrm{CH}_{3} \mathrm{CN}\right): \mathrm{C}, 68.82 ; \mathrm{H}, 7.90 ; \mathrm{N}, 6.59$. Found: $\mathrm{C}, 68.71 ; \mathrm{H}$, $7.63 ; \mathrm{N}, 6.24$.

Synthesis of RuPNN ${ }^{\text {imine }}$. In the glovebox, RuPNN ${ }^{\mathrm{HCl}}$ (207 mg, $0.42 \mathrm{mmol}$ ), tricyclohexylphosphine (142 $\mathrm{mg}, 0.51 \mathrm{mmol}$ ), and potassium $t$-butoxide $(95 \mathrm{mg}, 0.85 \mathrm{mmol}$ ) were added to a $10 \mathrm{~mL}$ vial along with a stirbar. Toluene $(1.5 \mathrm{~mL})$ was added, and the mixture was heated to $80^{\circ} \mathrm{C}$ for one hour, then to $100{ }^{\circ} \mathrm{C}$ for 20 minutes. The mixture was allowed to cool, and was filtered through a PTFE filter disk into a $20 \mathrm{~mL}$ vial, washing through with $1 \mathrm{~mL}$ toluene. The solution was layered with $15 \mathrm{~mL}$ acetonitrile, causing the slow formation of dark purple crystals. After two days, the supernatant was carefully decanted away, and the remaining solid was washed 10 times with a 4:1 mixture of acetonitrile and toluene. The dark purple, highly air-sensitive solid product was dried under vacuum and stored in the glovebox at room temperature. Yield: $190 \mathrm{mg}, 64 \% .{ }^{1} \mathrm{H}$ NMR $\left(\mathrm{C}_{6} \mathrm{D}_{6}, 400.13 \mathrm{MHz}, 22{ }^{\circ} \mathrm{C}\right): \delta 7.53\left(\mathrm{dd},{ }^{4} \mathrm{~J}_{\mathrm{HP}}=2.1,5.2 \mathrm{~Hz}, 1 \mathrm{H}\right.$, $\left.\mathrm{CH}_{\text {imine }}\right) ; 7.05\left(\mathrm{~d},{ }^{3} \mathrm{~J}_{\mathrm{HH}}=8.6 \mathrm{~Hz}, 1 \mathrm{H}, \mathrm{CH}_{\text {pyr }}\right) ; 6.61\left(\mathrm{ddd},{ }^{3} \mathrm{~J}_{\mathrm{HH}}=6.3,8.6 \mathrm{~Hz},{ }^{5} J_{\mathrm{HP}}=3.7 \mathrm{~Hz}, 1 \mathrm{H}, \mathrm{CH}_{\text {pyr }}\right) ; 6.30(\mathrm{~m}$, $1 \mathrm{H}, \mathrm{CH}_{\mathrm{pyr}}$ ); $4.57\left(\mathrm{dqd},{ }^{2} J_{\mathrm{HH}}=11.6 \mathrm{~Hz},{ }^{3} J_{\mathrm{HH}}=7.2 \mathrm{~Hz},{ }^{4} J_{\mathrm{HP}}=3.5 \mathrm{~Hz}, 1 \mathrm{H}, \mathrm{CH}_{2-\mathrm{Et}}\right) ; 3.95\left(\mathrm{dq},{ }^{2} J_{\mathrm{HH}}=11.6 \mathrm{~Hz},{ }^{3} J_{\mathrm{HH}}=\right.$ $7.2 \mathrm{~Hz}, 1 \mathrm{H}, \mathrm{CH}_{2-\mathrm{Et}}$ ); $3.68\left(\mathrm{~d},{ }^{2} J_{\mathrm{HH}}=16.25 \mathrm{~Hz}, 1 \mathrm{H}, \mathrm{CH}_{2} \mathrm{P}\right.$ ); $3.43\left(\mathrm{~d},{ }^{2} J_{\mathrm{HH}}=16.25 \mathrm{~Hz},{ }^{3} J_{\mathrm{HP}}=11.3 \mathrm{~Hz}, 1 \mathrm{H}, \mathrm{CH}_{2} \mathrm{P}\right.$ ); $2.1\left(\mathrm{br} \mathrm{d},{ }^{2} J_{\mathrm{HH}}=12.7 \mathrm{~Hz}, 3 \mathrm{H}, \mathrm{PCy}\right) ; 1.94-1.76(\mathrm{~m}, 6 \mathrm{H}, \mathrm{PCy}) ; 1.71\left(\mathrm{t},{ }^{3} \mathrm{~J}_{\mathrm{HH}}=7.2 \mathrm{~Hz}, 3 \mathrm{H}, \mathrm{CH}_{3-\mathrm{Et}}\right) ; 1.73-1.43$ $\left(\mathrm{m}, 12 \mathrm{H}, \mathrm{PC} y_{3}\right) ; 1.32\left(\mathrm{~d},{ }^{3} \mathrm{~J}_{\mathrm{HP}}=12.3 \mathrm{~Hz}, 9 \mathrm{H}, \mathrm{CH}_{3-\mathrm{tBu}}\right) ; 1.27-1.02(\mathrm{~m}, 12 \mathrm{H}, \mathrm{PCy}) ; 0.91\left(\mathrm{~d},{ }^{3} \mathrm{~J}_{\mathrm{HP}}=12.1 \mathrm{~Hz}, 9 \mathrm{H}\right.$, $\left.\mathrm{CH}_{3-\mathrm{tBu}}\right) .{ }^{13} \mathrm{C}\left\{{ }^{1} \mathrm{H}\right\} \operatorname{NMR}\left(\mathrm{C}_{6} \mathrm{D}_{6}, 100.62 \mathrm{MHz}, 22^{\circ} \mathrm{C}\right): \delta 218.4\left(\mathrm{dd},{ }^{2} \mathrm{~J}_{\mathrm{CP}}=7.7,13.8 \mathrm{~Hz}, \mathrm{CO}\right) ; 158.2\left(\mathrm{t},{ }^{2,4} J_{\mathrm{CP}}=5.4\right.$ $\mathrm{Hz}, \mathrm{C}_{\text {pyr }}$ ); 144.4 (d, ${ }^{3} \mathrm{~J}_{\mathrm{CP}}=4.0 \mathrm{~Hz}, \mathrm{C}_{\mathrm{pyr}}$ ); 135.9 ( $\left.\mathrm{C}_{\text {imine }}\right) ; 121.0$ (d, $\left.{ }^{5} \mathrm{~J}_{\mathrm{CP}}=2.8 \mathrm{~Hz}, \mathrm{C}_{\mathrm{pyr}}\right) ; 120.1$ (d, ${ }^{4} \mathrm{~J}_{\mathrm{CP}}=3.7 \mathrm{~Hz}$, $\left.\mathrm{C}_{\mathrm{pyr}}\right) ; 105.6\left(\mathrm{dd},{ }^{3} \mathrm{~J}_{\mathrm{CP}}=10.6 \mathrm{~Hz},{ }^{4} \mathrm{~J}_{\mathrm{CP}}=4.1 \mathrm{~Hz}, \mathrm{C}_{\mathrm{pyr}}\right) ; 61.1\left(\mathrm{~d},{ }^{2} J_{\mathrm{CP}}=5.1 \mathrm{~Hz}, \mathrm{CH}_{2-\mathrm{Et}}\right) ; 41.8\left(\mathrm{~d},{ }^{1} J_{\mathrm{CP}}=12.0 \mathrm{~Hz}, \mathrm{C}_{\mathrm{tBu}}\right)$; 
$41.0\left(d,{ }^{1} J_{C P}=9.0 \mathrm{~Hz}, P C y_{3}\right) ; 36.8\left(d,{ }^{1} J_{C P}=14.2 \mathrm{~Hz}, C_{2} P\right) ; 33.6\left(d,{ }^{1} J_{C P}=5.6 \mathrm{~Hz}, C_{t B u}\right) ; 30.4\left(d,{ }^{2} J_{C P}=6.4 \mathrm{~Hz}\right.$, $\left.\mathrm{C}_{\mathrm{tBu}}\right) ; 29.9\left(\mathrm{PCY}_{3}\right) ; 29.4\left(\mathrm{PCY}_{3}\right) ; 29.0\left(\mathrm{~d},{ }^{2} J_{\mathrm{CP}}=4.7 \mathrm{~Hz}, \mathrm{C}_{\mathrm{tBu}}\right) ; 28.0\left(\mathrm{~d},{ }^{2} J_{\mathrm{CP}}=9.5 \mathrm{~Hz}, \mathrm{PCY}\right) ; 27.9\left(\mathrm{~d},{ }^{2} J_{\mathrm{HH}}=9.8\right.$ $\left.\left.\mathrm{Hz}, \mathrm{PC} y_{3}\right) ; 26.9\left(\mathrm{PC}_{3}\right) ; 20.6\left(\mathrm{~d},{ }^{4} \mathrm{~J}_{\mathrm{CP}}=7.4 \mathrm{~Hz}, \mathrm{CH}_{2} \mathrm{CH}_{3}\right) .{ }^{31} \mathrm{P}\left\{{ }^{1} \mathrm{H}\right\} \mathrm{NMR} \mathrm{C}_{6} \mathrm{D}_{6}, 101.97 \mathrm{MHz}, 22{ }^{\circ} \mathrm{C}\right): \delta 113.58(\mathrm{~d}$, $\left.{ }^{2} J_{\mathrm{PP}}=10.7 \mathrm{~Hz}, \mathrm{P}^{\mathrm{t} B u}\right) ; 54.51\left(\mathrm{~d},{ }^{2} \mathrm{~J}_{\mathrm{PP}}=10.7 \mathrm{~Hz}, \mathrm{PC} y_{3}\right)$. Anal calcd. For $\mathrm{C}_{36} \mathrm{H}_{62} \mathrm{~N}_{2} \mathrm{OP}_{2} \mathrm{Ru}: \mathrm{C}, 61.60 ; \mathrm{H}, 8.90 ; \mathrm{N}$, 3.99. Found: $C, 61.95 ; H, 9.09 ; N, 4.02$.

Synthesis and VT-NMR Studies of RuPNN ${ }^{\text {HEt }}$. In the glovebox, a sample of RuPNN ${ }^{\text {imine }}(0.3 \mathrm{mg}, 0.4 \mu \mathrm{mol})$ was dissolved in $0.20 \mathrm{~mL}$ toluene- $\mathrm{d}_{8}$ and transferred to a Wilmad Heavy Wall Pressure/Vacuum NMR Tube, part no. 522-PV-7. The tube was closed and brought out of the box. It was then pressurized with 10 bar $\mathrm{H}_{2}$ and vented three times, inverted several times to saturate the solution, then pressurized and vented three more times. The dark purple solution became colorless over approximately 20 minutes, after which time NMR spectra were recorded. ${ }^{1} \mathrm{H}$ NMR spectra were recorded every $10{ }^{\circ} \mathrm{C}$ between -70 and $+60{ }^{\circ} \mathrm{C}$, at which point decomposition began to occur. COSY, NOESY, and ${ }^{31} \mathrm{P}\left\{{ }^{1} \mathrm{H}\right\}$ NMR spectra were recorded at $-30^{\circ} \mathrm{C}$. Upon evaporation of the solvent and redissolution in toluene- $\mathrm{d}_{8}$, a purple solution was observed, which was confirmed by ${ }^{1} \mathrm{H}$ NMR to contain RuPNN ${ }^{\text {imine }}$, demonstrating the reversibility of the reaction at room temperature. ${ }^{1} \mathrm{H} N M R\left(C_{7} D_{8}, 400.13 \mathrm{MHz},-30{ }^{\circ} \mathrm{C}\right): \delta 6.67\left(\mathrm{t},{ }^{3} \mathrm{~J}_{\mathrm{HH}}=7.7 \mathrm{~Hz}, 1 \mathrm{H}, \mathrm{CH}_{\mathrm{pyr}}\right)$; $6.40\left(\mathrm{~d},{ }^{3} \mathrm{~J}_{\mathrm{HH}}=7.7 \mathrm{~Hz}, 1 \mathrm{H}, \mathrm{CH}_{\text {pyr }}\right) ; 6.26\left(\mathrm{~d},{ }^{3} \mathrm{~J}_{\mathrm{HH}}=7.7 \mathrm{~Hz}, 1 \mathrm{H}, \mathrm{CH}_{\mathrm{pyr}}\right) ; 5.89(\mathrm{br}, 1 \mathrm{H}, \mathrm{NH}) ; 4.61\left(\mathrm{br} \mathrm{d},{ }^{2} \mathrm{~J}_{\mathrm{HH}}=15.2\right.$ $\mathrm{Hz}, 1 \mathrm{H}, \mathrm{CH}_{2} \mathrm{NEt}$ ); 3.77 (d, ${ }^{2} J_{\mathrm{HH}}=15.2 \mathrm{~Hz}, 1 \mathrm{H}, \mathrm{CH}_{2} \mathrm{NEt}$ ); 2.91 (d, $\left.{ }^{2} J_{\mathrm{HP}}=8.6 \mathrm{~Hz}, 2 \mathrm{H}, \mathrm{CH}_{2} \mathrm{P}\right) ; 2.70(\mathrm{~m}, 2 \mathrm{H}$, $\left.\mathrm{NCH}_{2} \mathrm{CH}_{3}\right) ; 1.40\left(\mathrm{~d},{ }^{3} J_{\mathrm{HP}}=12.7 \mathrm{~Hz}, 9 \mathrm{H}, \mathrm{P}^{\mathrm{t} B u}\right) ; 1.33\left(\mathrm{~d},{ }^{3} J_{\mathrm{HP}}=12.5 \mathrm{~Hz}, 9 \mathrm{H}, \mathrm{P}^{\mathrm{t}} \mathrm{Bu}\right) ;-4.42\left(\mathrm{~d},{ }^{2} \mathrm{~J}_{\mathrm{HP}}=13.8 \mathrm{~Hz}, 1 \mathrm{H}\right.$, $\mathrm{RuH}) ;-5.09\left(\mathrm{~d},{ }^{2} J_{\mathrm{HP}}=17.5 \mathrm{~Hz}, 1 \mathrm{H}, \mathrm{RuH}\right)$. The $\mathrm{NCH}_{2} \mathrm{CH}_{3}$ resonance is obscured beneath the $\mathrm{P}^{\mathrm{t}} \mathrm{Bu}$ and free $\mathrm{PCy}_{3}$ resonances at $-30{ }^{\circ} \mathrm{C}$, but is visible in the range of $-60{ }^{\circ} \mathrm{C}$ to $-40{ }^{\circ} \mathrm{C}$ as a triplet with ${ }^{3} \mathrm{JHH}_{\mathrm{HH}}=6.5 \mathrm{~Hz}$. Its chemical shift at $-40{ }^{\circ} \mathrm{C}$ is $1.51 \mathrm{ppm} .{ }^{31} \mathrm{P}\left\{{ }^{1} \mathrm{H}\right\} \mathrm{NMR}\left(\mathrm{C}_{7} \mathrm{D}_{8}, 101.97 \mathrm{MHz},-30{ }^{\circ} \mathrm{C}\right): \delta 120.55$.

X-ray Crystallography. For all three crystal structures reported herein, X-ray quality crystals were grown by layering acetonitrile over a concentrated toluene solution. Structure determinations were performed on an Oxford Diffraction Gemini-R diffractometer, using Mo-Ka radiation at $110 \mathrm{~K}$ unless otherwise noted. Crystals were mounted on Hampton Research Cryoloops using Paratone-N oil. Unit cell determination, data collection and reduction, and empirical absorption correction were performed using the CrysAlisPro software package. ${ }^{4}$ Direct methods structure solution was accomplished using SIR92, ${ }^{5}$ and full-matrix least-squares refinement was carried out using CRYSTALS. ${ }^{6}$ All non-hydrogen atoms were refined anisotropically. Hydrogen atoms were placed in calculated positions, and their positions were initially refined using distance and angle restraints. All hydrogen positions were fixed in place for the final refinement cycles.

Apparatus for Time-Course Monitoring of Hydrogenation Reactions. Time-course monitoring of hydrogenation reactions was conducted using an Asynt Multicell Parallel High Pressure Reactor, customized to fit in the glovebox antechamber and to include reaction sampling valves on five reactor cells. The sampling valves employed $0.8 \mathrm{~mm}$ ID 1/16" stainless steel tubing, a Swagelok Low Flow metering valve (part no. SS-SS1) to control flow, and a Swagelok ball valve (part no. SS-41GS1) to allow removal of samples. Longer or shorter lengths of tubing allowed sampling of the liquid phase or the headspace. As the internal volume of the sampling valve system was measured to be $0.25 \mathrm{~mL}$, removal 
of $0.50 \mathrm{~mL}$ of liquid before collecting each aliquot ensured that a fresh sample was being taken directly from the reaction mixture. A sixth reactor cell was fitted with a thermocouple to allow control of the internal reaction temperatures. For heated reactions, the reactor was partially submerged $(2 \mathrm{~cm}$ past the internal liquid level) in a stirred oil bath (a non-magnetic stainless-steel cooking pot), whose temperature was controlled using heating tape wrapped around the oil bath and an Omega Platinum Series Universal Benchtop Digital Controller, part no. CS8DPT. The oil bath was placed on a stirring hotplate, which allowed stirring of the reactions and oil bath, as well as pre-heating of the oil to allow the reactions to equilibrate to the desired temperature quickly.

Experimental Procedure for Time-Course Monitoring of Hydrogenation Reactions. First, the oil bath was preheated to an empirically determined temperature above the desired reaction temperature to enable rapid heating to the desired internal temperature $\left(138^{\circ} \mathrm{C}\right.$ for reactions at $105^{\circ} \mathrm{C}$, and $103^{\circ} \mathrm{C}$ for reactions at $85^{\circ} \mathrm{C}$ ). The Asynt reactor was brought into the glovebox, along with oven-dried glass liners and stir bars. Inside the glovebox, reaction solutions were prepared with the desired amount of ester (hexyl hexanoate in this work), catalyst, and 0.20 equiv. of tetradecane relative to ester as an internal standard. The total liquid reaction volume was $10.0 \mathrm{~mL}$. The reactor was sealed and brought out of the glovebox. After gently purging the $\mathrm{H}_{2}$ line for 3 minutes, the reactor was pressurized to 20 bar and vented three times, then filled to 20 bar a fourth time. The thermocouple was connected to the Omega temperature controller, and the reactor was submerged in the pre-heated oil bath. The internal reaction temperature was recorded each minute until it was stable, and time zero was marked when the temperature was $5{ }^{\circ} \mathrm{C}$ below the target temperature. Typically, the warmup time was 5-10 minutes. An example temperature curve is shown in Figure $\mathrm{S} 1$.

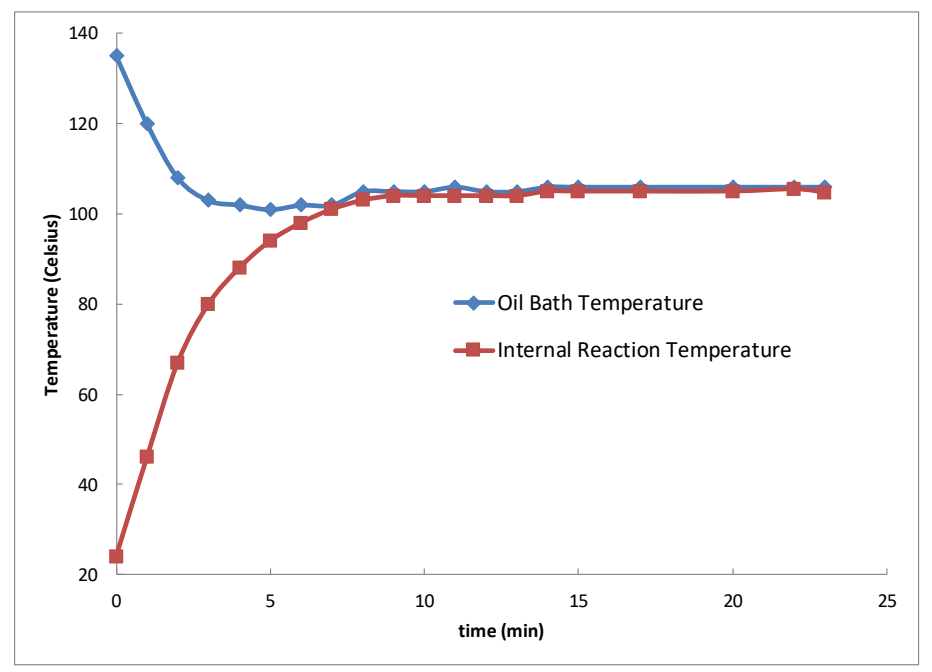

Figure S1. Temperature of oil bath and internal reaction temperature during a typical time-course monitoring experiment.

Measurement of Ethane and Propane Formation During Hydrogenation Reactions. Reactions were assembled and conducted in the Asynt Multicell Reactor as described above, using a sampling tube that extended into the headspace of the reaction rather than the liquid phase. Experiments were not 
conducted in parallel to avoid the possibility of gas transfer between reaction wells. Outside of the reactor, the sampling tubing was run into a glass recrystallization dish filled with water, and gas samples were collected using GC vials initially filled with water, which were inverted and placed over the sampling tube to collect gas, and subsequently capped underwater. For each aliquot, a $5 \mathrm{~mL}$ vial was first filled with gas which was discarded, as the $\sim 0.25 \mathrm{~mL}$ internal volume of the sampling valve expands by 20 -fold after decompression from 20 bar to atmospheric pressure. Then, a $1.5 \mathrm{~mL} \mathrm{GC}$ vial was filled with gas and analyzed directly by GC-FID, with an injection volume of $5 \mu \mathrm{L}$ (an autosampler made the injections, the volumes of which are precise within 1\%). Ethane and propane were detected using a 25 meter Agilent $\mathrm{CP}-\mathrm{Al}_{2} \mathrm{O}_{3} / \mathrm{Na}_{2} \mathrm{SO}_{4}$ column, part no. $\mathrm{CP} 7586$. The $\mathrm{GC}$ method ran for 3.0 minutes at $50{ }^{\circ} \mathrm{C}$. Ethane eluted with a retention time of 1.12 minutes and propane eluted at 1.84 minutes. The Airgas UHP hydrogen used contained a small amount of methane (retention time 1.02 minutes) and ethane (comprising about $5 \%$ of the maximum signal observed in our experiments). No concentration standard was used in these measurements: the graphs in Figure 4 represent the absolute FID signal, with the hydrogen's ethane impurity subtracted and then normalized so that the largest value is equal to 1 .

Hydrogenation Substrate Scope Experiments in a Stainless-Steel Pressure Reactor. In the glovebox, the catalyst, ester, and 0.20 equiv. tetradecane as internal standard were dissolved in the appropriate solvent and transferred to a test tube with a stir bar. All substrates except for phthalide were soluble in the initial reaction solution. The tube was placed in a stainless-steel pressure reactor, which was sealed and brought out of the glovebox. The reactor was pressurized with $30 \mathrm{bar} \mathrm{H}_{2}$ and vented three times, and was pressurized a fourth time to 30 bar. The reactor was placed on a stir plate in a water bath set to $25{ }^{\circ} \mathrm{C}$, and stirred for 16 hours. The reactor was carefully vented and opened to air. An aliquot of the reaction mixture was taken and analyzed by GC-FID. Yields were calculated based on the calibrated response factors of the products relative to the tetradecane standard. Reactions of achiral substrates were analyzed by GC-FID on a 15 meter Shimadzu SH-Rxi-1MS column. The reaction of (S)-ethyl ibuprofen was analyzed using a 30 meter Agilent Cyclosil-B column, with a temperature ramp from 130 to $165^{\circ} \mathrm{C}$ over 35 minutes.

Computational Details. Density functional theory calculations were performed using the Gaussian 16 computational chemistry package, Revision B.01. ${ }^{7}$ The geometries and energies of all species were calculated using the hybrid functional B3LYP, ${ }^{8}$ augmented with the addition of empirical dispersion with Grimme's D3 dispersion corrections ${ }^{9}$ (referred to as B3LYP-d3). Ru was modeled with the effective core potential of Hay and Wadt ${ }^{10}$ and the accompanying uncontracted basis set (including $f$ polarization functions $)^{11}$ collectively known as LANL08(f). ${ }^{12}$ All other elements were modeled with the 6-311G(d,p) basis set. ${ }^{13}$ Complete structures with no truncations were used in all cases, and were optimized in solvent, using a polarizable continuum with radii and non-electrostatic terms from Truhlar and coworkers' SMD solvation model, and with dielectric constants chosen for toluene. ${ }^{14}$ Frequency calculations ensured the absence of imaginary vibrational modes. Standard state corrections were added in order to adjust from $1 \mathrm{~atm}$ to $1 \mathrm{M}$ for solution-phase free energies, amounting to $1.89 \mathrm{kcal} / \mathrm{mol}$ added to the free energy of each isolated molecule at $298.15 \mathrm{~K} .{ }^{15}$ The solvation-corrected electronic energies were further refined using the M06 functional ${ }^{16}$ and the same basis sets described above. 
Energies are given below in Tables S1 and S2, and geometries in Cartesian coordinates are included in a separate, compiled .XYZ file.

Table S1. Energies of isolated species calculated by DFT.

\begin{tabular}{lll} 
Compound & Electronic energy (hartrees) & Free energy correction (hartrees) \\
\hline $\mathrm{H}_{2}$ & -1.170093135 & -0.00144 \\
$\mathrm{PC}_{3}$ & -1046.79222 & 0.432459 \\
RuPNN $^{\text {imine }}$ & -2369.828105 & 0.845781 \\
RuPNN $^{\text {HEt }}$ & -1325.388578 & 0.426134 \\
RuCNN-Et $^{\text {imine }}$ & -2519.415215 & 0.926228 \\
RuCNN $^{\text {HEt }}$ & -1474.97103 & 0.502946
\end{tabular}

Table S2. Energies with mass balance.

\begin{tabular}{llll} 
Compound(s) & $\begin{array}{l}\text { Electronic Energy } \\
\text { (hartrees) }\end{array}$ & $\begin{array}{l}\text { Free energy correction, } \\
298.15 \mathrm{~K} \text { (hartrees) }\end{array}$ & $\begin{array}{l}\text { Relative free energy at } 1 \mathrm{M} \text { std. } \\
\text { state }(\mathrm{kcal} / \mathrm{mol}), 298.15 \mathrm{~K}\end{array}$ \\
\hline RuPNN $^{\text {imine }}+2 \mathrm{H}_{2}$ & -2372.168291 & 0.842901 & 0.00 \\
RuPNN $^{\text {HEt }}+\mathrm{PCY}_{3}$ & -2372.180798 & 0.858593 & +0.11 \\
RuCNN-Et $^{\text {imine }}+2 \mathrm{H}_{2}$ & -2521.755401 & 0.923348 & 0.00 \\
RuCNN $^{\text {HEt }}+\mathrm{PCY}_{3}$ & -2521.76325 & 0.935405 & +0.75
\end{tabular}




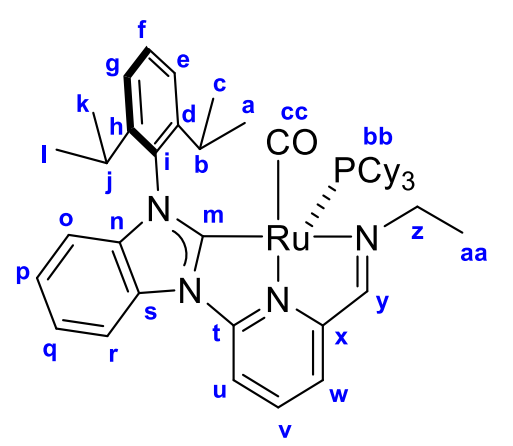

${ }^{*} \mathrm{MeCN}$

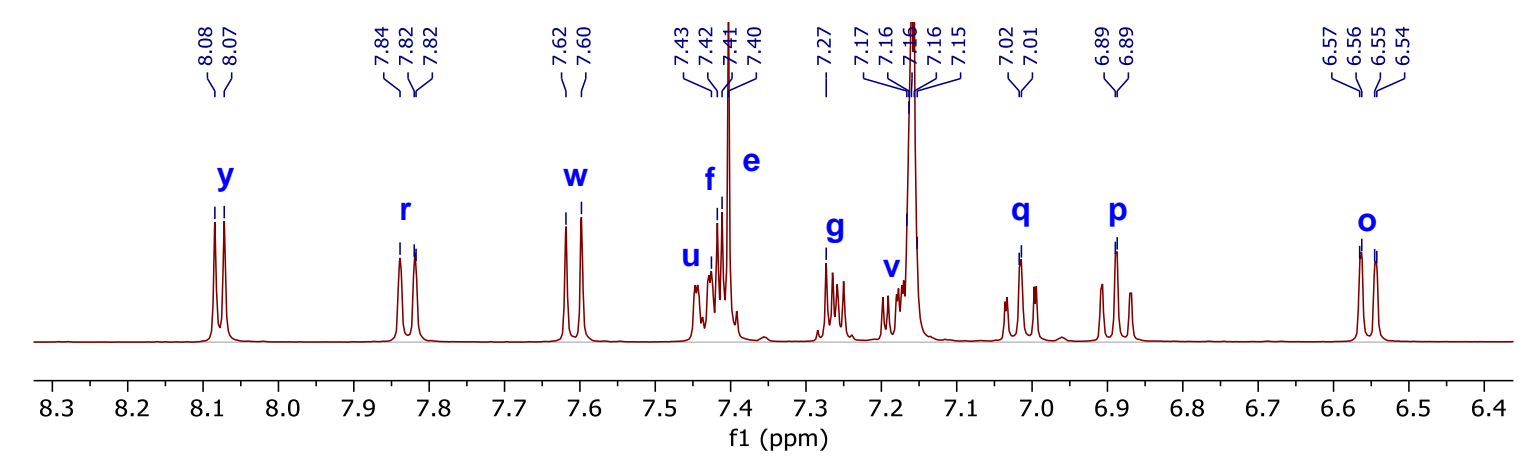

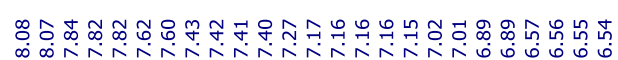
U

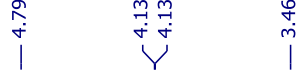

I

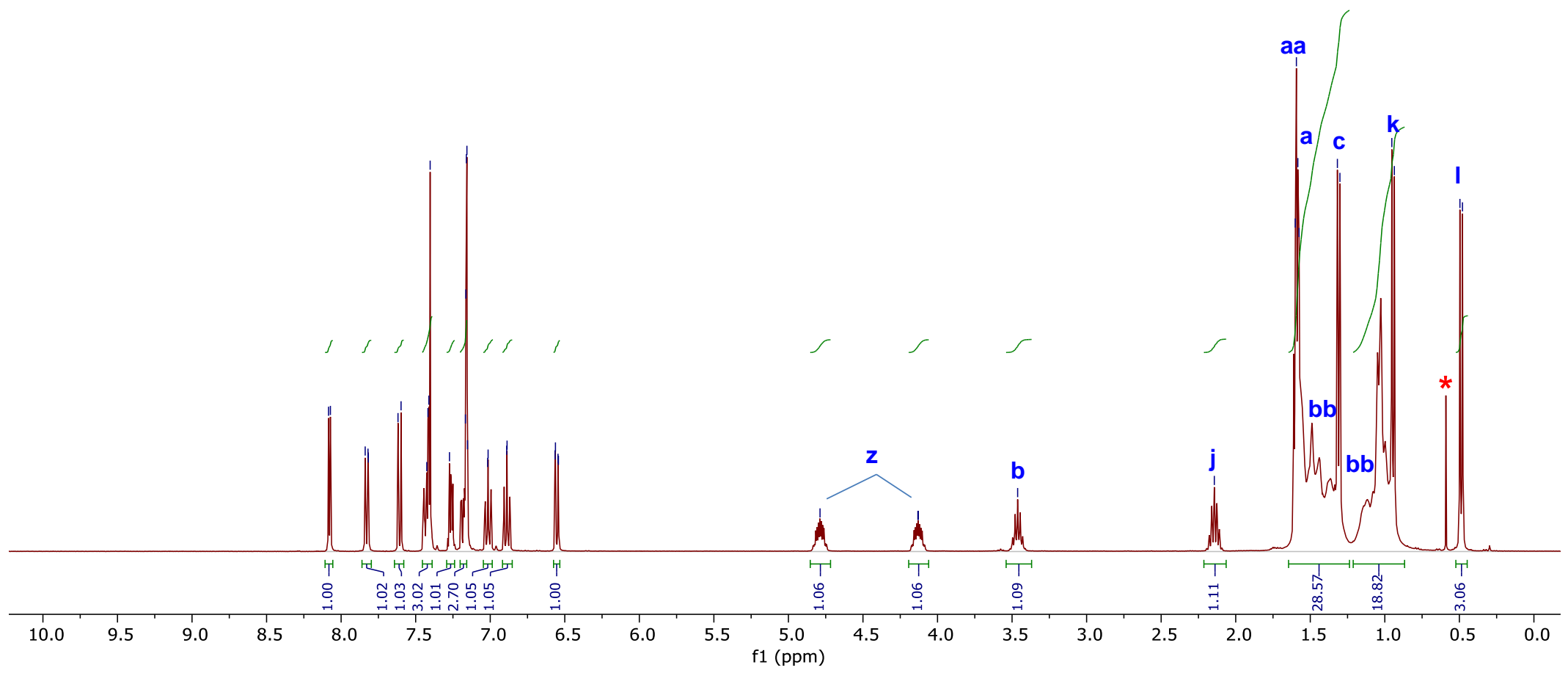

Figure S2. ${ }^{1} \mathrm{H} N \mathrm{NR}\left(\mathrm{rt}, \mathrm{C}_{6} \mathrm{D}_{6}, 400.13 \mathrm{MHz}\right)$ of RuCNN-Et ${ }^{\text {imine }}$. 


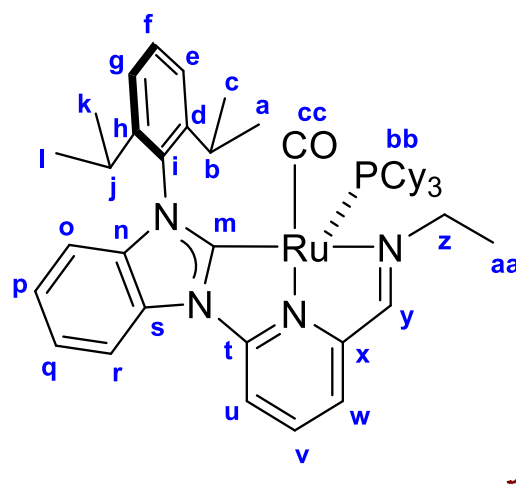

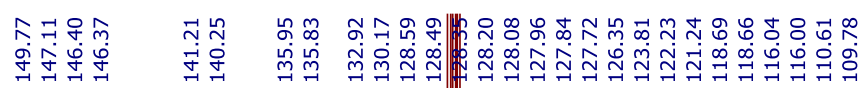

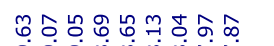
กกำกำ

। $\rightarrow>1 / 1$

in

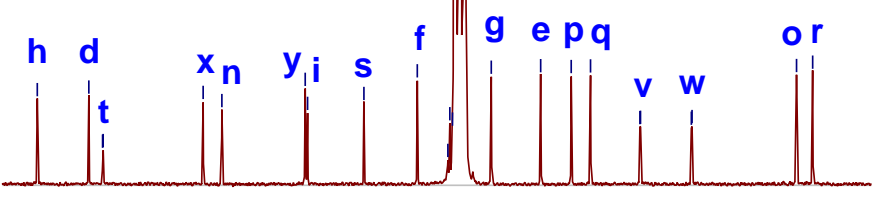

bb

$b, j \quad b b$

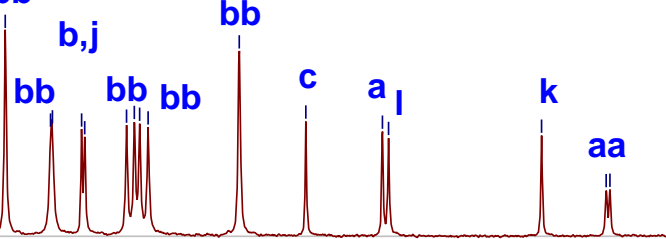

150

140

130125

120
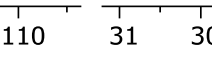

29 $27 \stackrel{26}{21}$

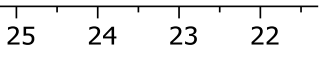

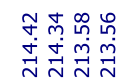

두웅

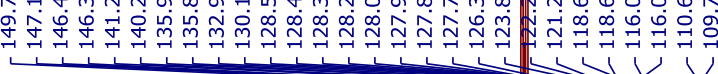

$\sqrt{4}$

$\stackrel{\substack{++}}{+}$

$\infty$
$\infty$
0
0
0
0
$Y$

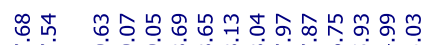

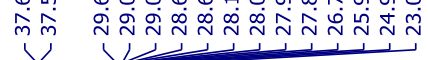

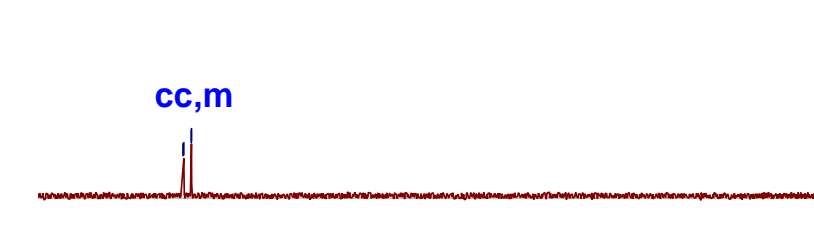

$190 \quad 180$

$\begin{array}{rr}120 & 110 \\ \mathrm{f} 1(\mathrm{ppm})\end{array}$

$\mathbf{u}$

Figure S3. ${ }^{13} \mathrm{C}\left\{{ }^{1} \mathrm{H}\right\}$ NMR $\left(r t, \mathrm{C}_{6} \mathrm{D}_{6}, 100.62 \mathrm{MHz}\right)$ of RuCNN-Et imine 


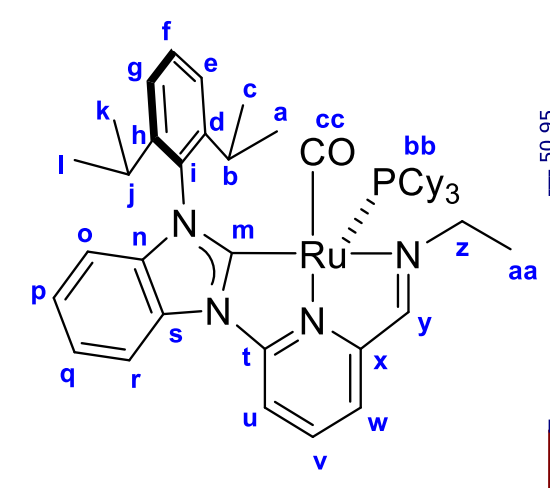

Figure S4. ${ }^{31} \mathrm{P}\left\{{ }^{1} \mathrm{H}\right\} \mathrm{NMR}\left(\mathrm{rt}, \mathrm{C}_{6} \mathrm{D}_{6}, 161.97 \mathrm{MHz}\right)$ of RuCNN-Et $^{\text {imine }}$. 


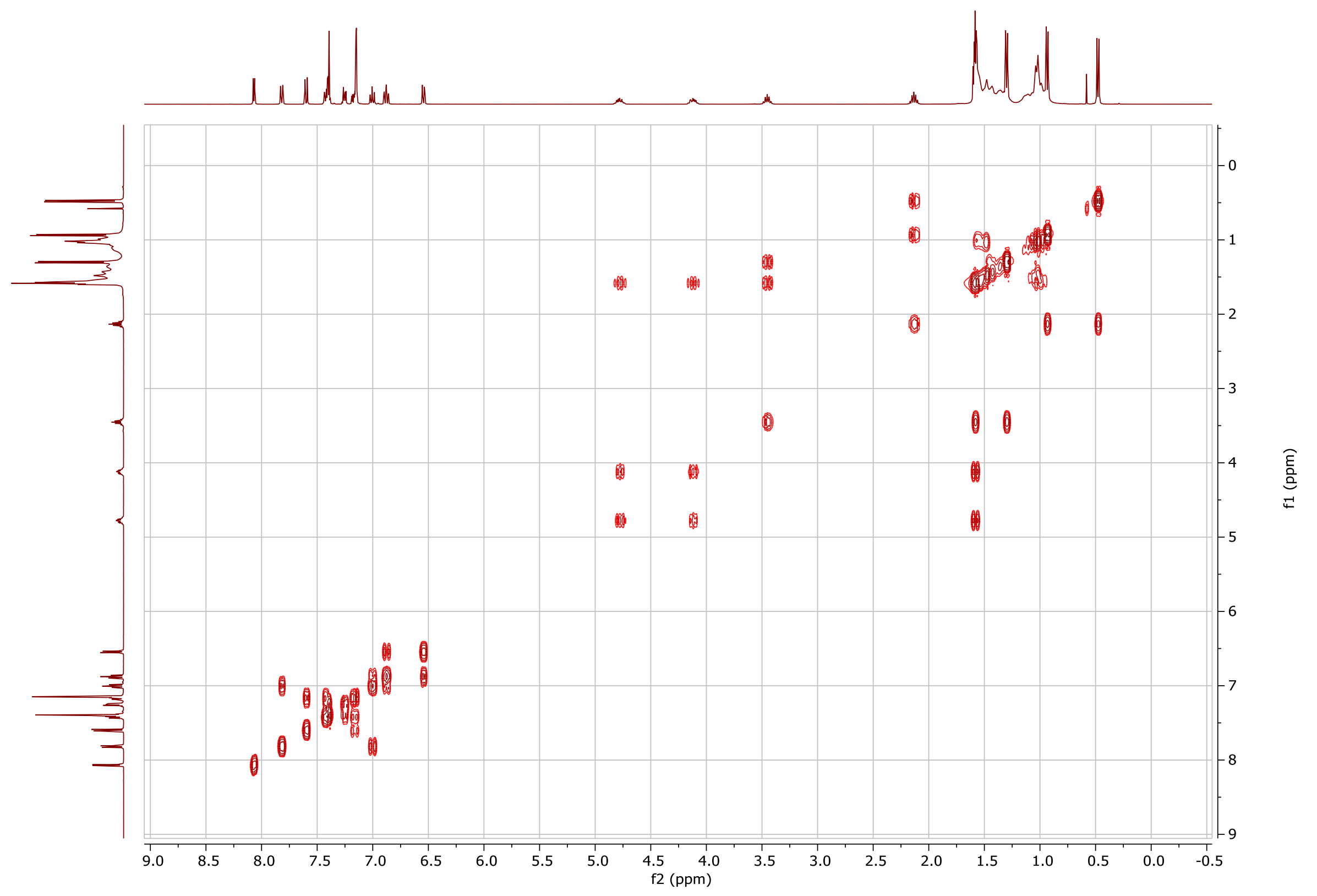

Figure S5. $\operatorname{COSY}\left(r t, \mathrm{C}_{6} \mathrm{D}_{6}\right)$ of RuCNN-Et ${ }^{\mathrm{imine}}$. 


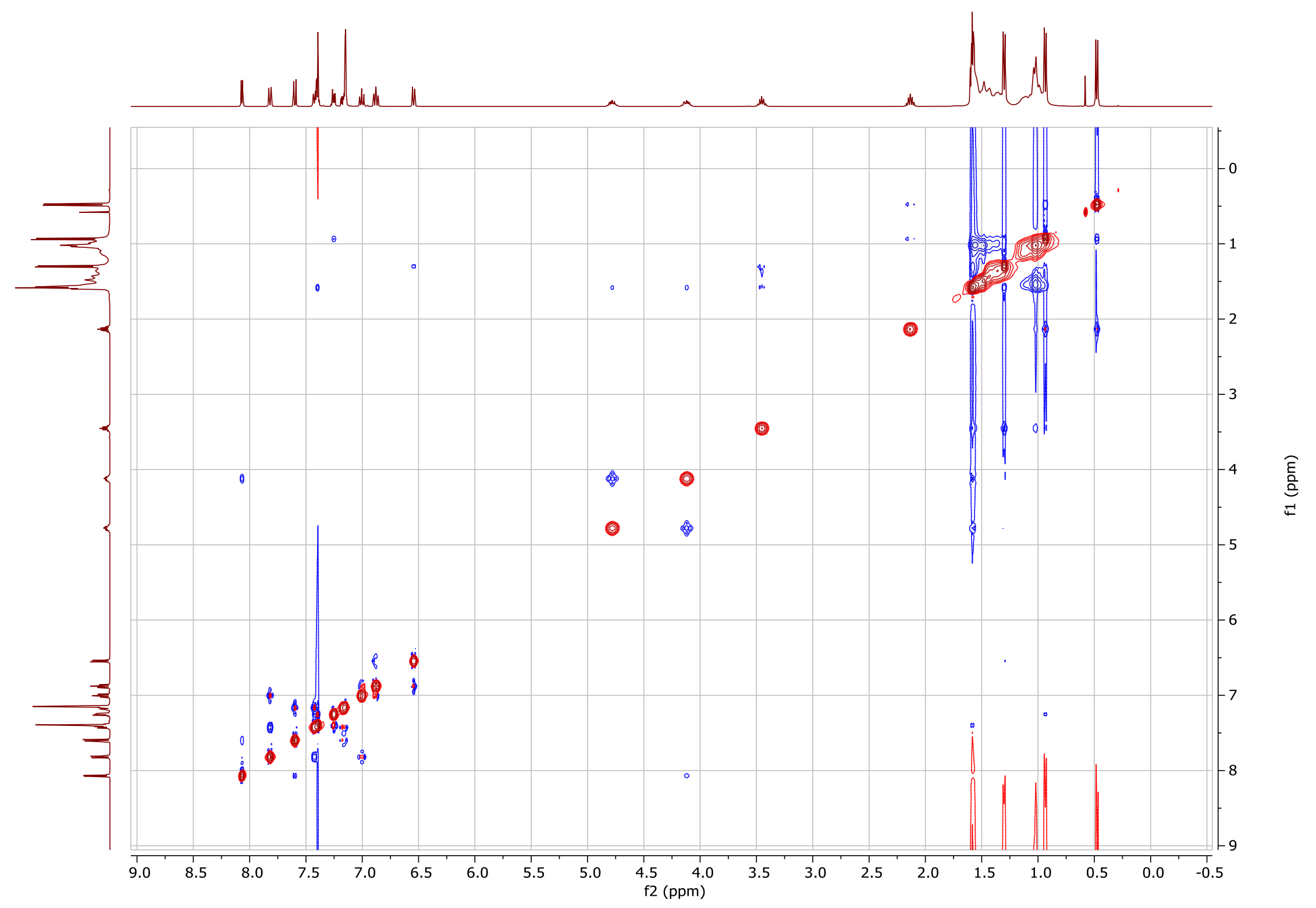

Figure S6. NOESY (rt, $\left.\mathrm{C}_{6} \mathrm{D}_{6}\right)$ of RuCNN-Et ${ }^{\text {imine }}$. 


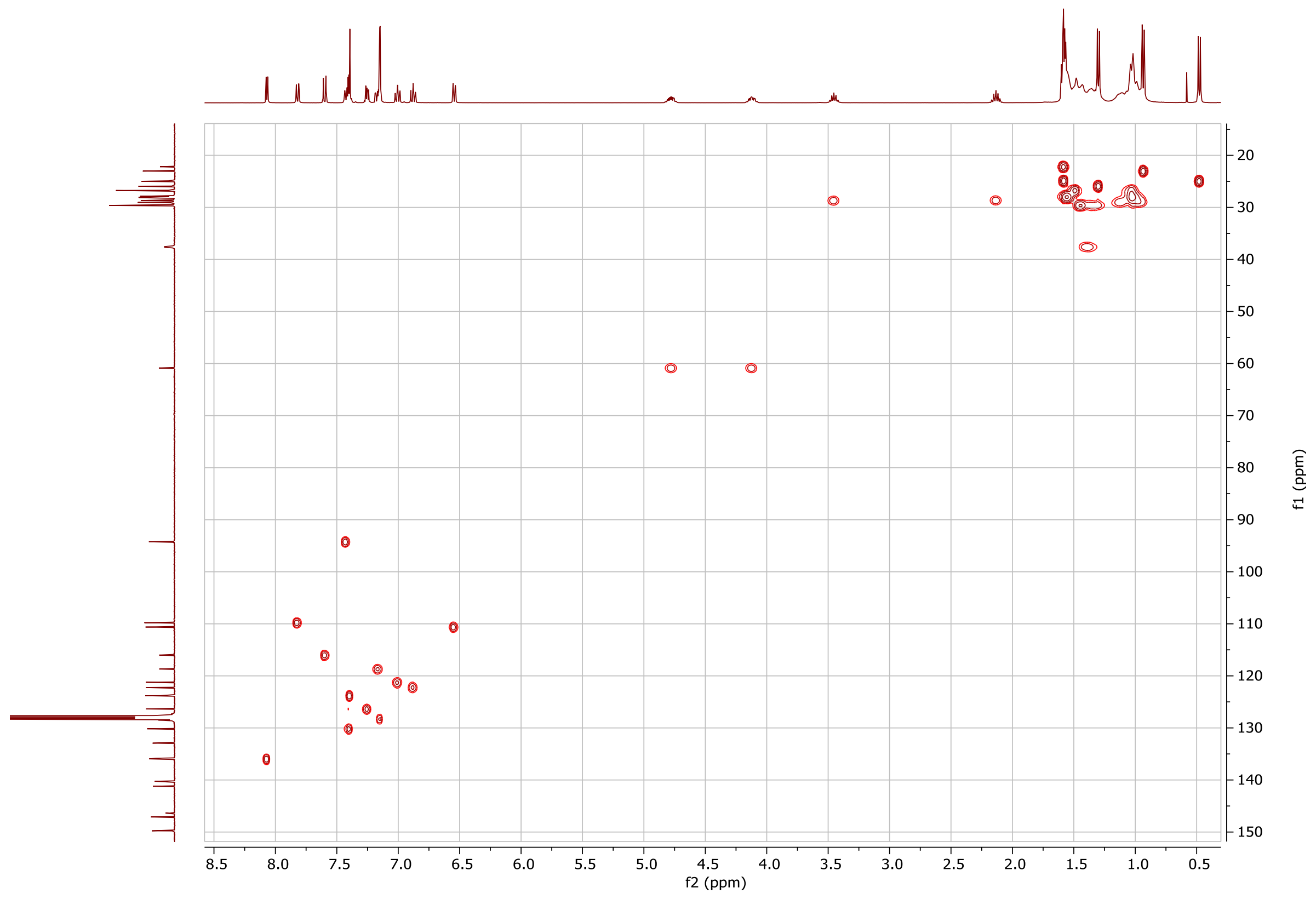

Figure S7. HSQC $\left(r t, C_{6} D_{6}\right)$ of RuCNN-Et ${ }^{i m i n e}$. 


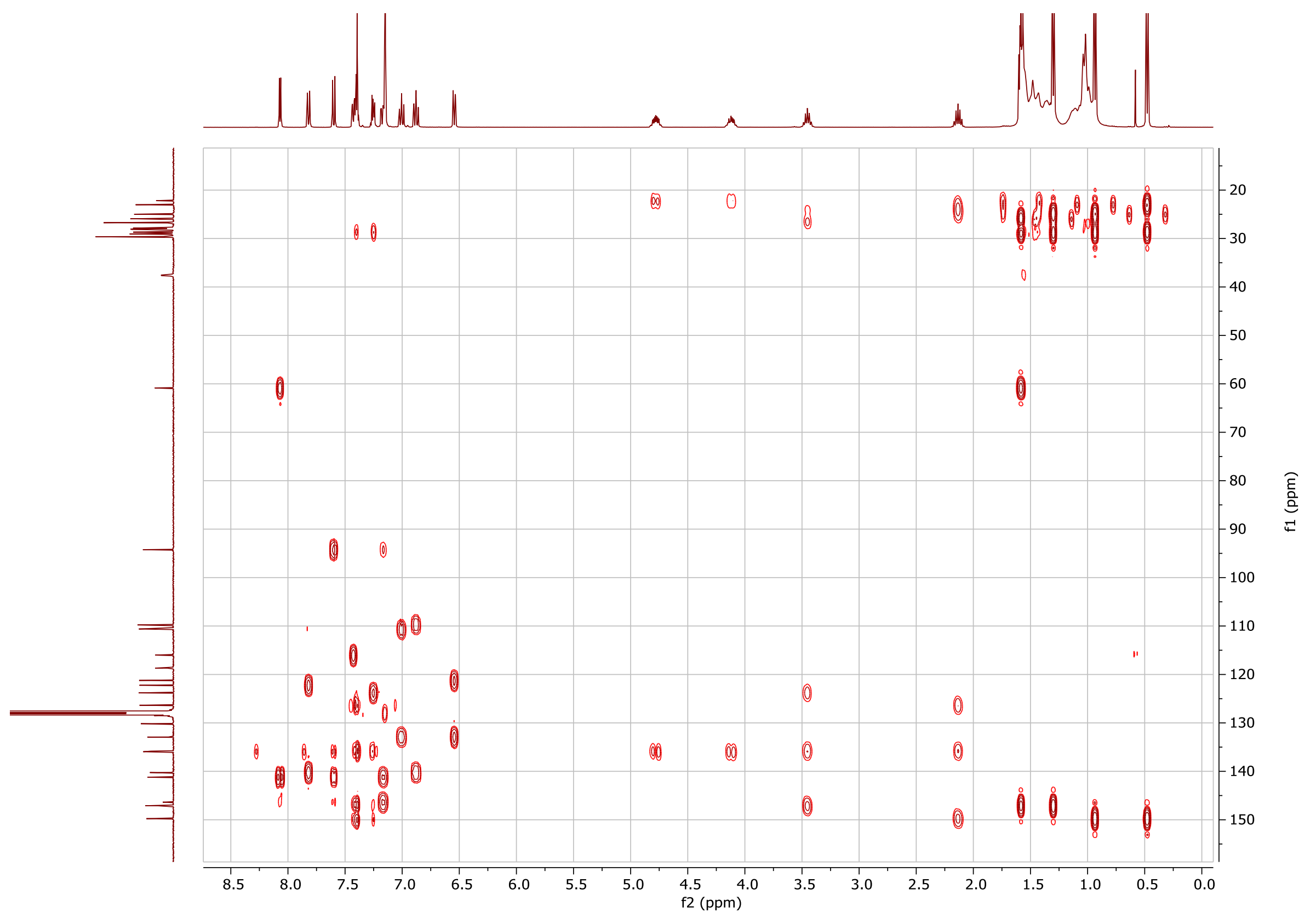

Figure S8. $H M B C\left(r t, C_{6} D_{6}\right)$ of RuCNN-Et ${ }^{i m i n e}$. 


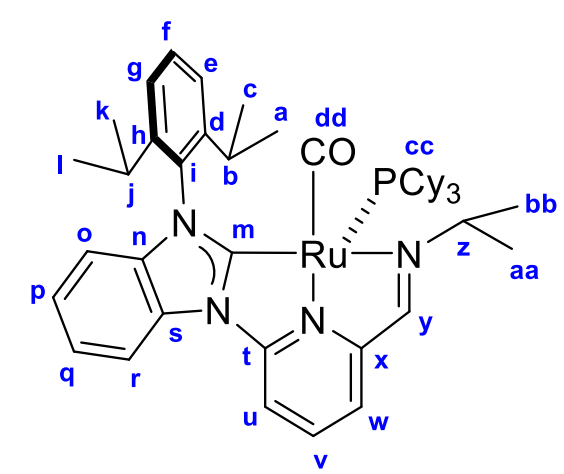

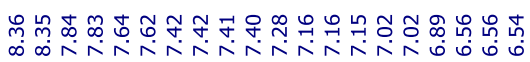
$\underbrace{1}$
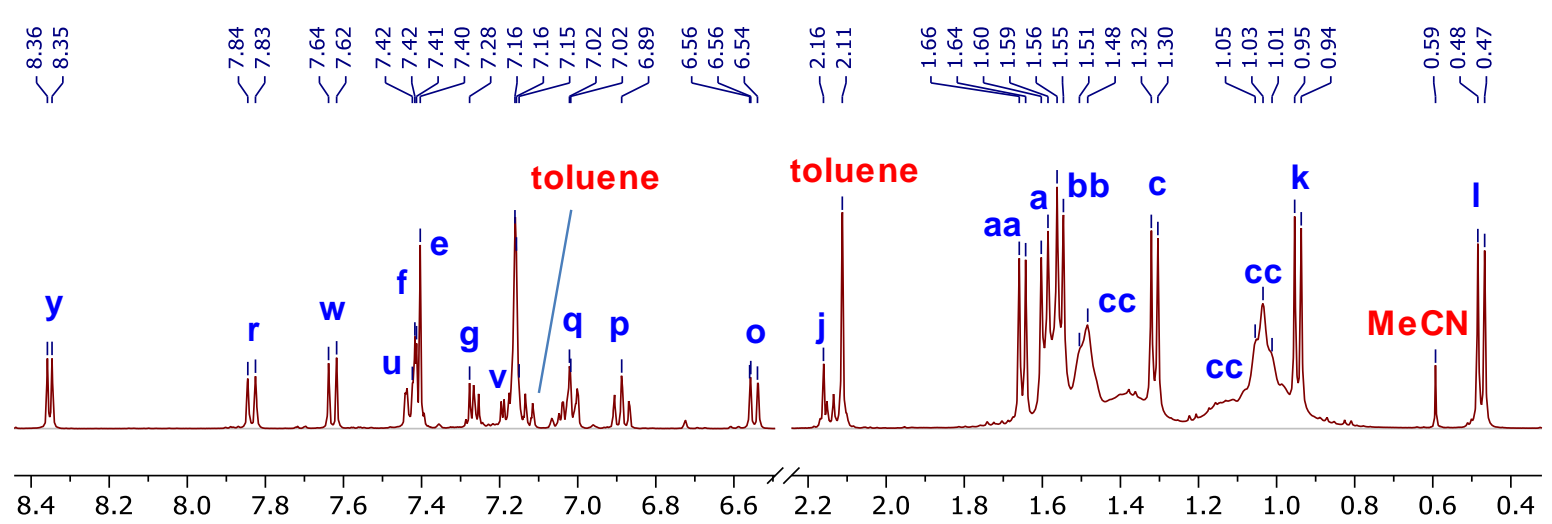
f1 (ppm)

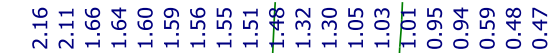

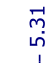

$\stackrel{\substack{+m}}{i}$

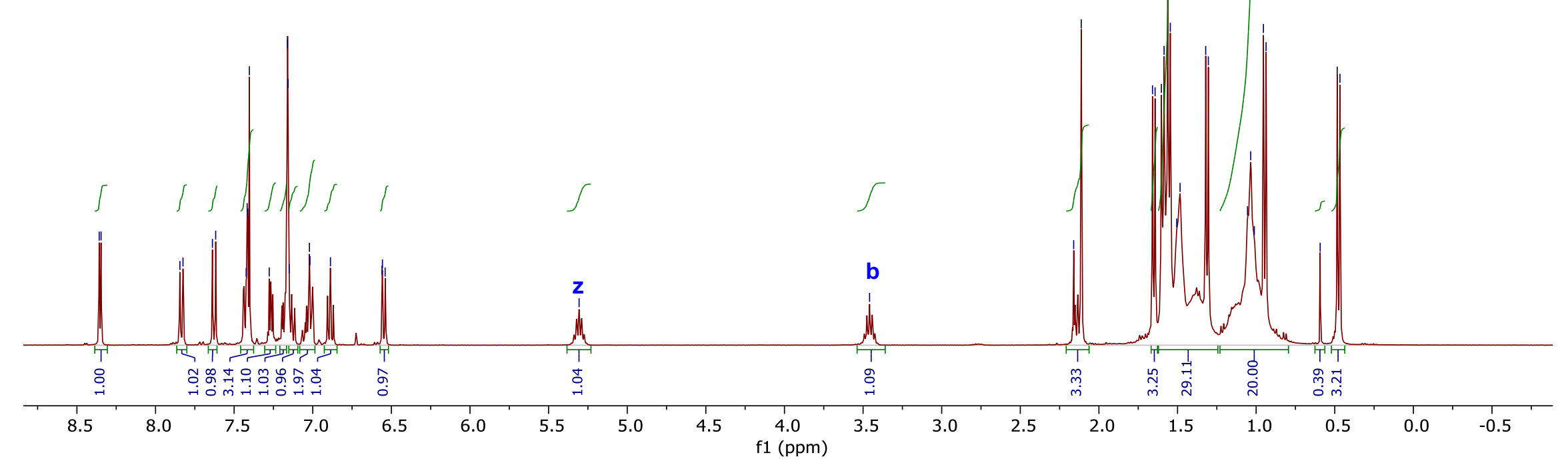

Figure S9. ${ }^{1} \mathrm{H} N M R\left(r t, C_{6} D_{6}, 400.13 \mathrm{MHz}\right.$ ) of RuCNN-'Primine. 


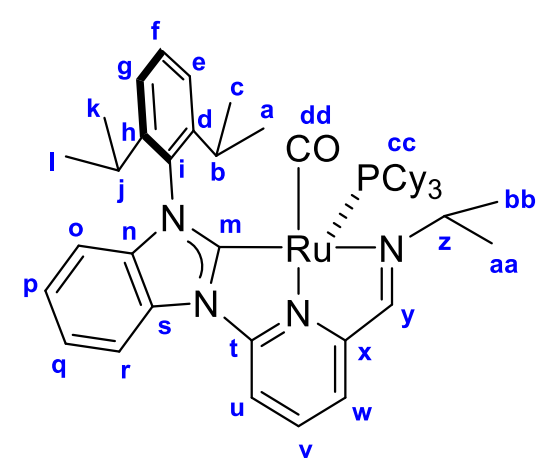

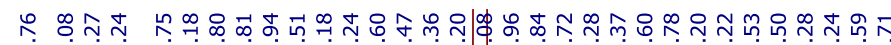

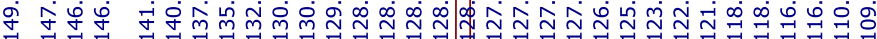

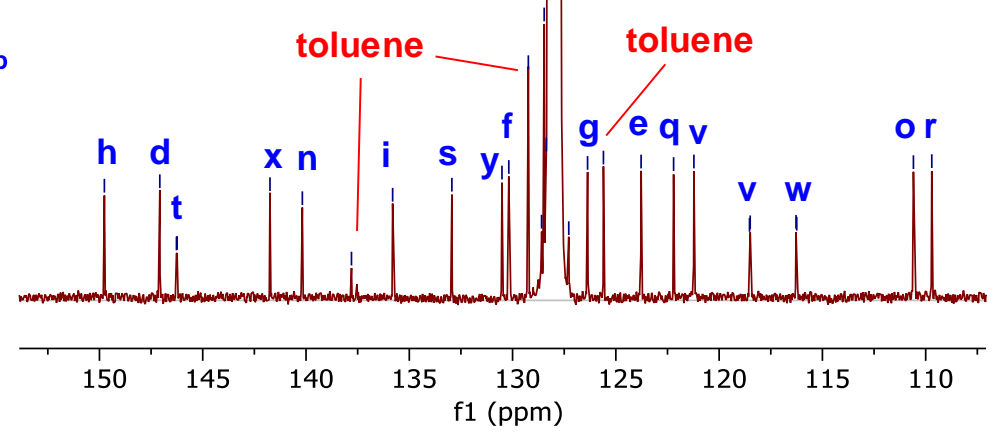

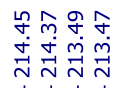

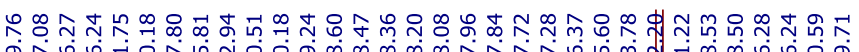

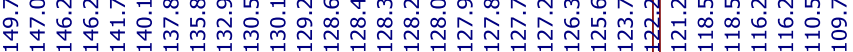

$\sqrt{4}$

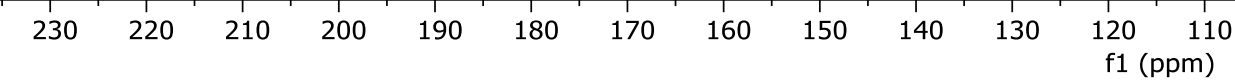

Figure S10. ${ }^{13} \mathrm{C}\left\{{ }^{1} \mathrm{H}\right\} \mathrm{NMR}\left(\mathrm{rt}, \mathrm{C}_{6} \mathrm{D}_{6}, 100.62 \mathrm{MHz}\right)$ of RuCNN-'Primine 


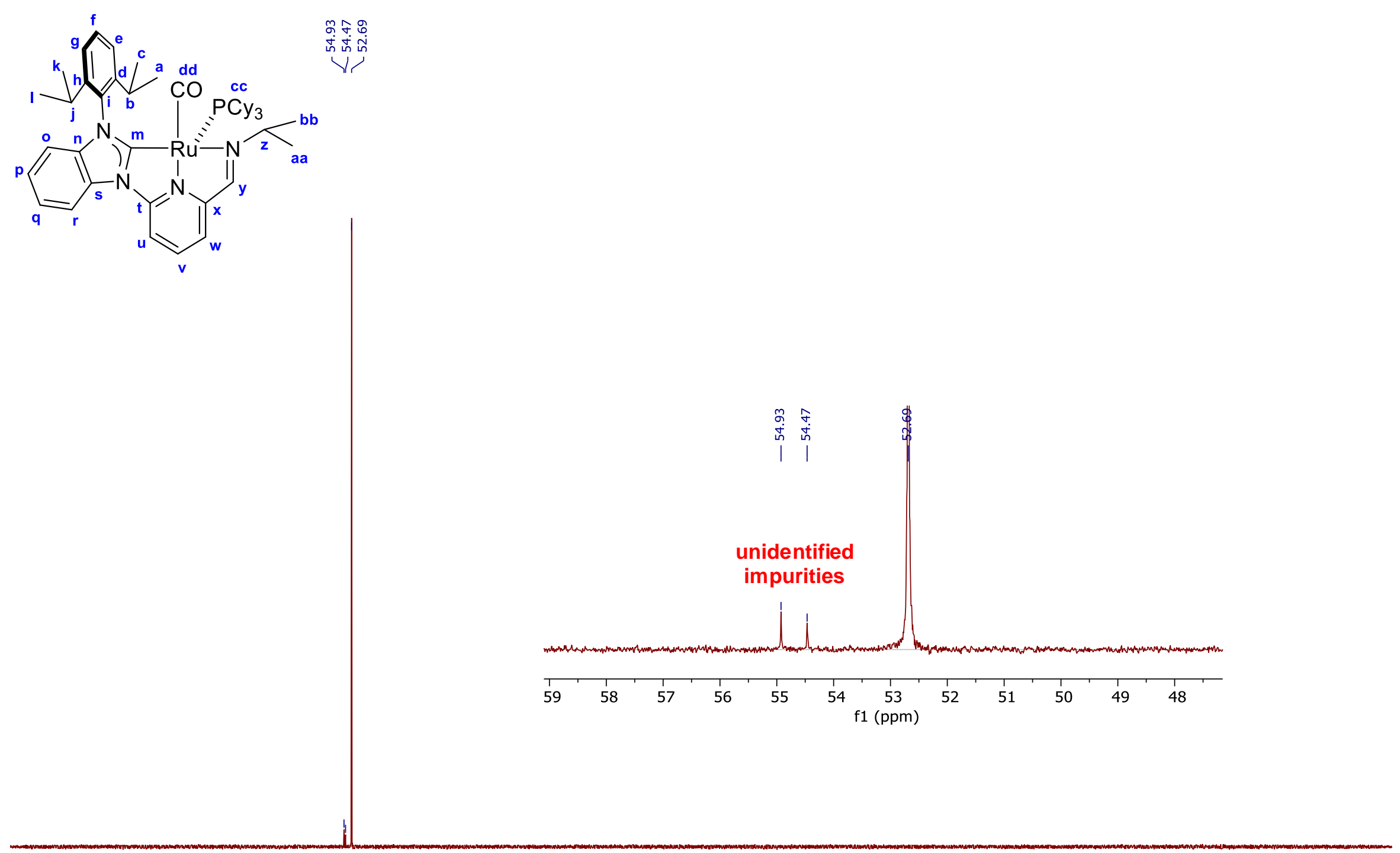

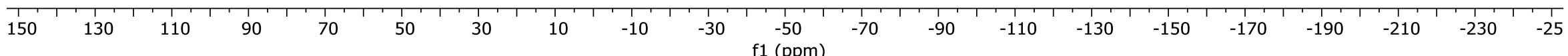

Figure S11. ${ }^{31} \mathrm{P}\left\{{ }^{1} \mathrm{H}\right\}$ NMR (rt, $\left.\mathrm{C}_{6} \mathrm{D}_{6}, 161.97 \mathrm{MHz}\right)$ of RuCNN-'Primine 


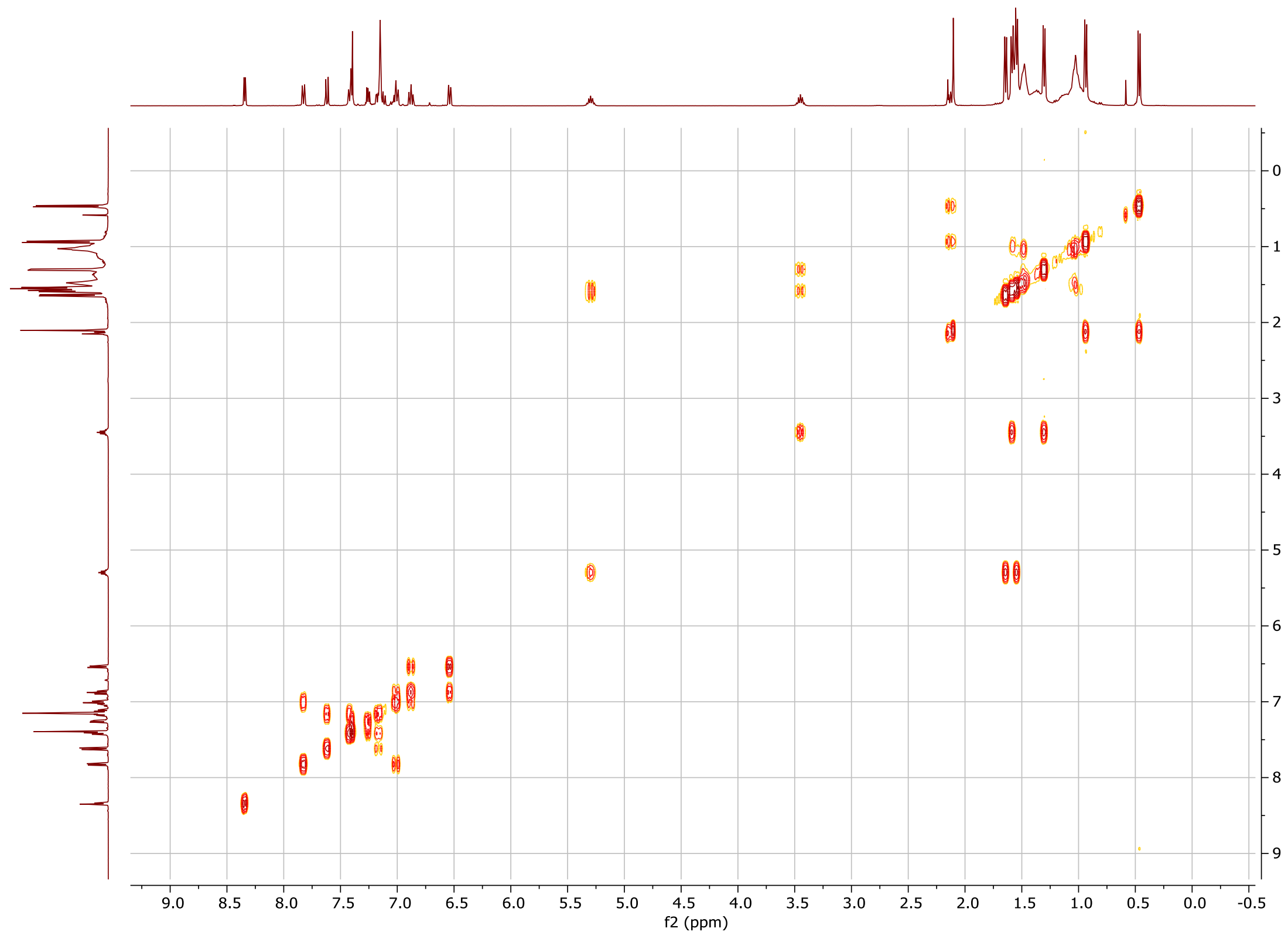

Figure S12. $\operatorname{COSY}\left(r t, C_{6} D_{6}\right)$ of RuCNN-'Primine. 


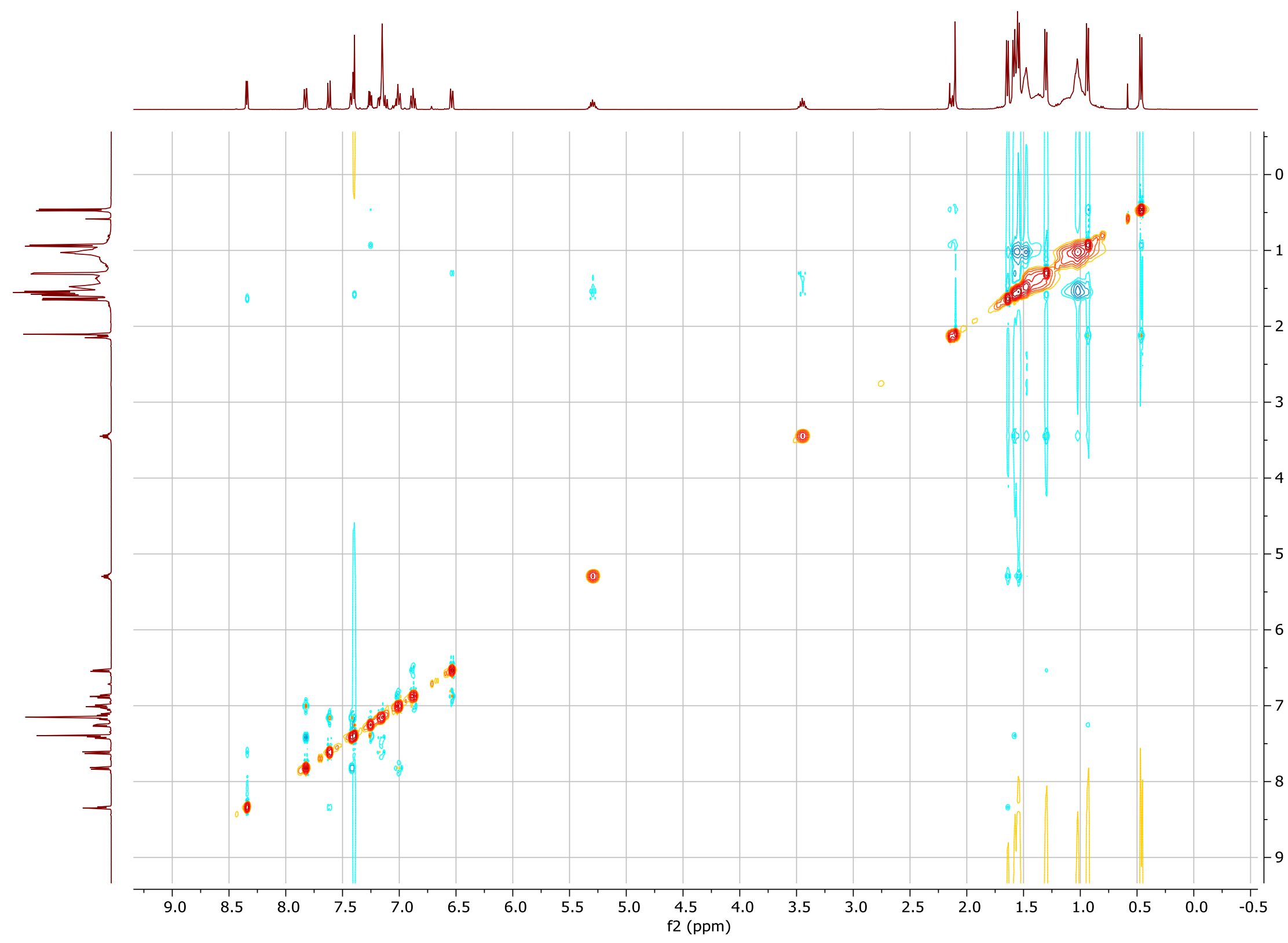

Figure S13. NOESY (rt, $\left.C_{6} D_{6}\right)$ of RuCNN-'Primine . 


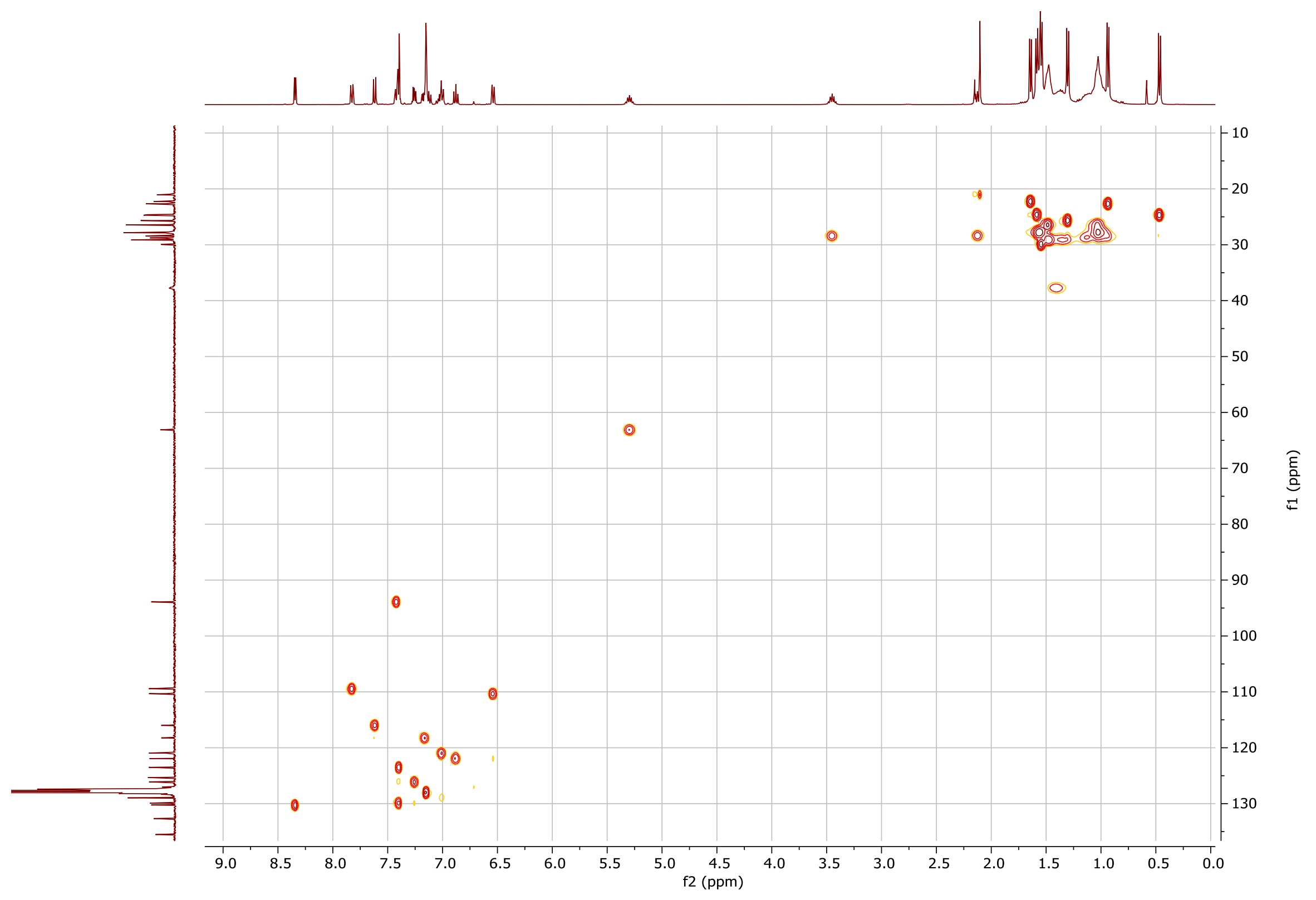

Figure S14. $\mathrm{HSQC}\left(\mathrm{rt}, \mathrm{C}_{6} \mathrm{D}_{6}\right)$ of RuCNN-'Primine . 


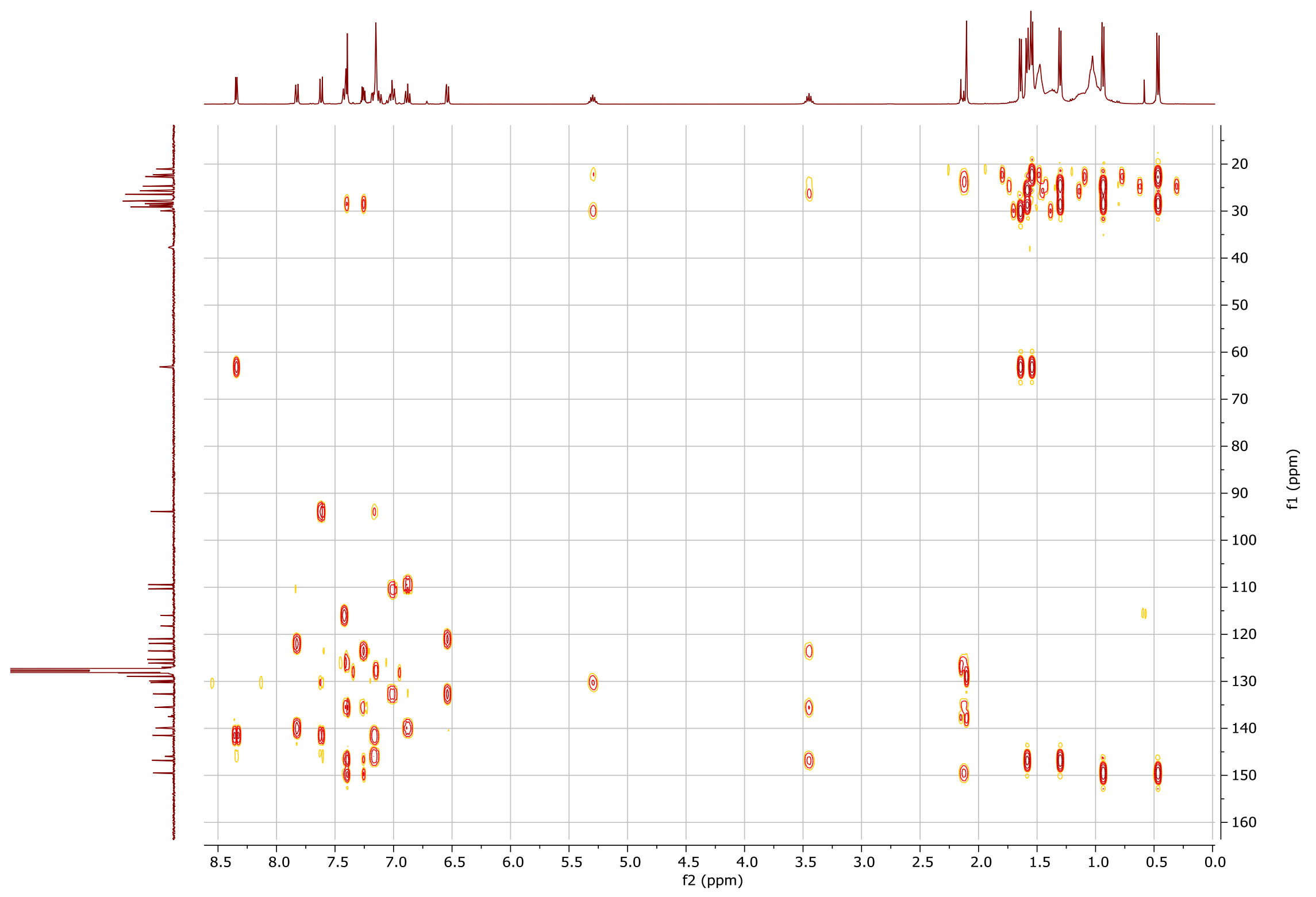

Figure S15. $\mathrm{HMBC}\left(r t, \mathrm{C}_{6} \mathrm{D}_{6}\right)$ of RuCNN-'Primine 

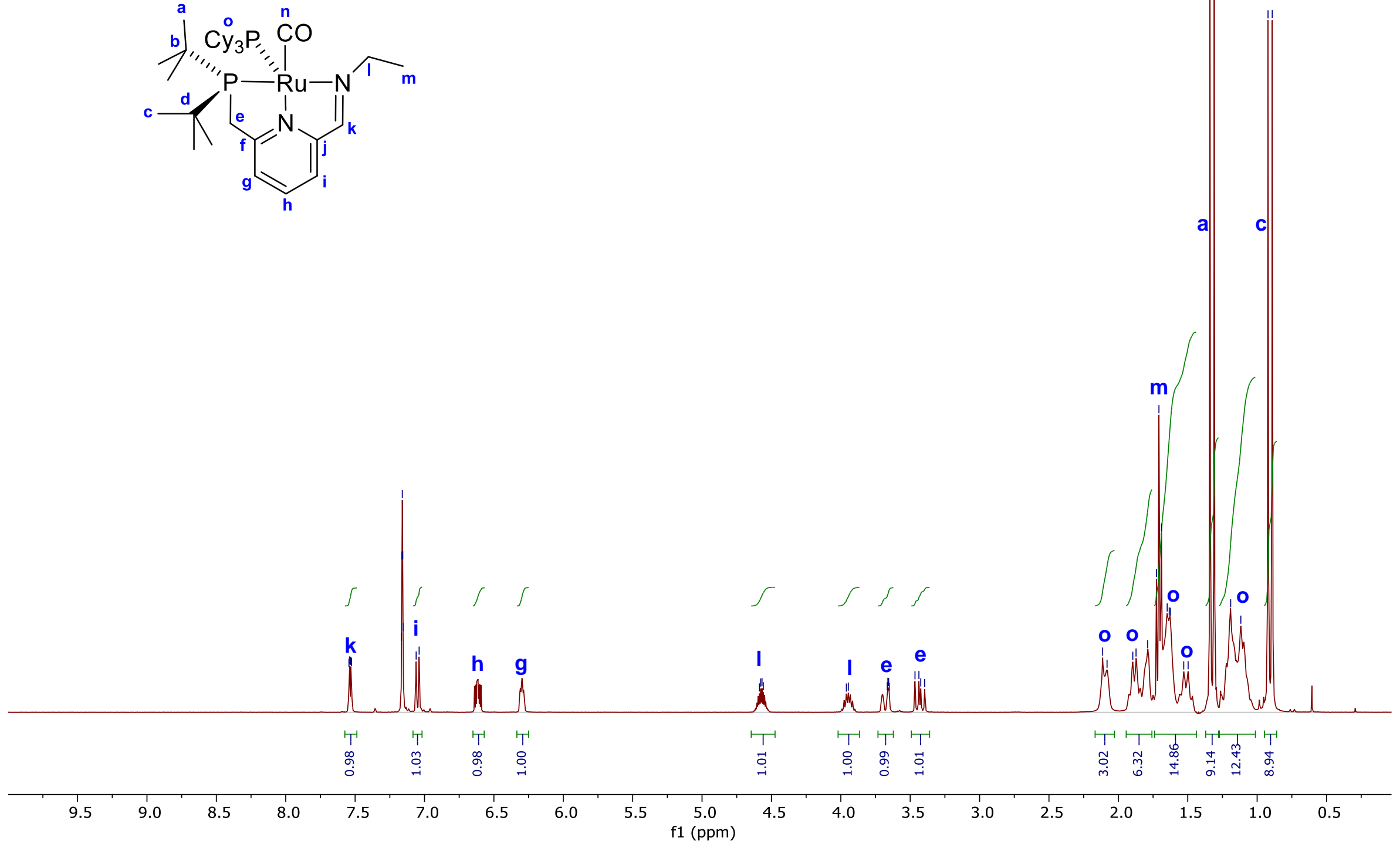

Figure S16. ${ }^{1} \mathrm{H}$ NMR $\left(r t, \mathrm{C}_{6} \mathrm{D}_{6}, 400.13 \mathrm{MHz}\right)$ of RuPNN ${ }^{\text {imine }}$. 

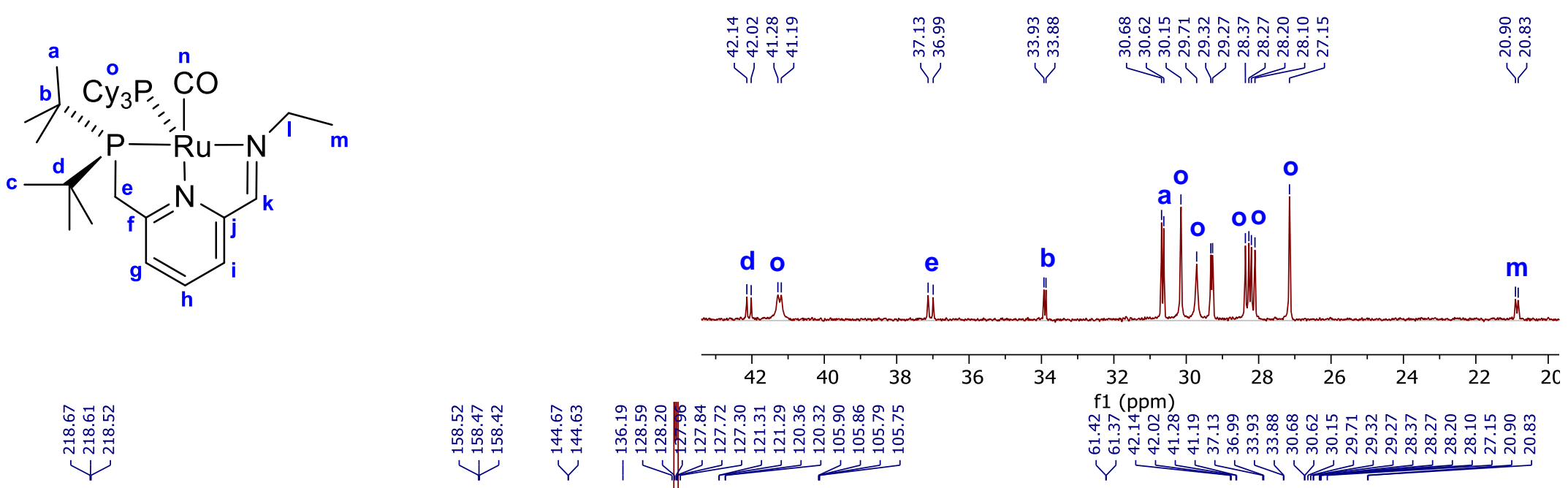

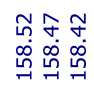

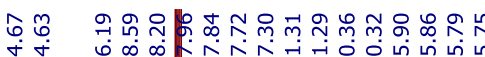

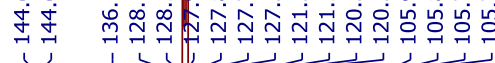

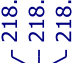

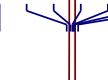

n

$250 \quad 240 \quad 230 \quad 220$

210200
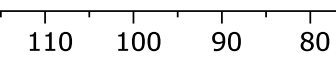

70

Figure S17. ${ }^{13} \mathrm{C}\left\{{ }^{1} \mathrm{H}\right\} \mathrm{NMR}\left(\mathrm{rt}, \mathrm{C}_{6} \mathrm{D}_{6}, 100.62 \mathrm{MHz}\right)$ of RuPNN ${ }^{\text {imine }}$. 

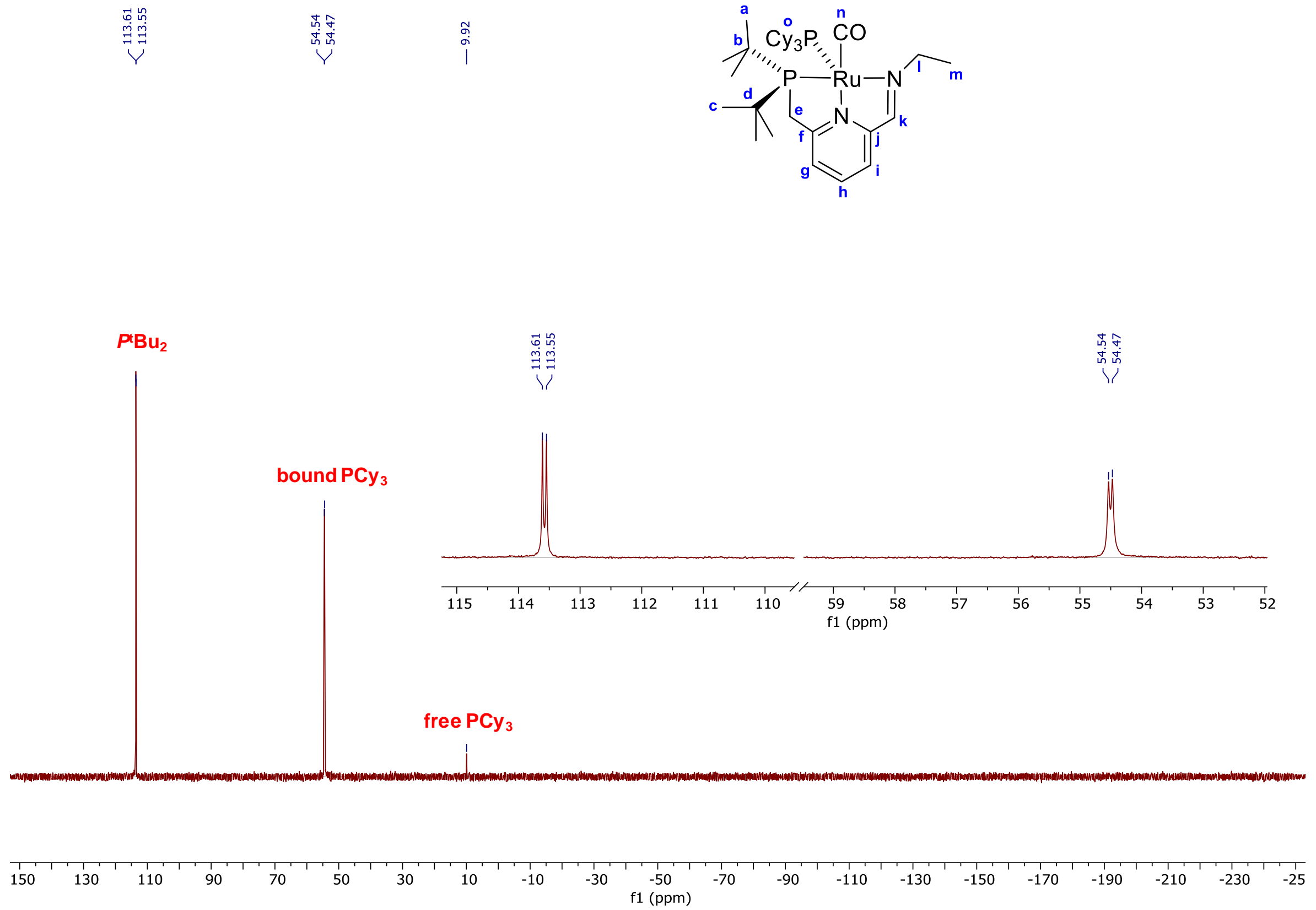

Figure S18. ${ }^{31} \mathrm{P}\left\{{ }^{1} \mathrm{H}\right\} \mathrm{NMR}\left(\mathrm{rt}, \mathrm{C}_{6} \mathrm{D}_{6}, 161.97 \mathrm{MHz}\right)$ of RuPNN ${ }^{\text {imine }}$. 


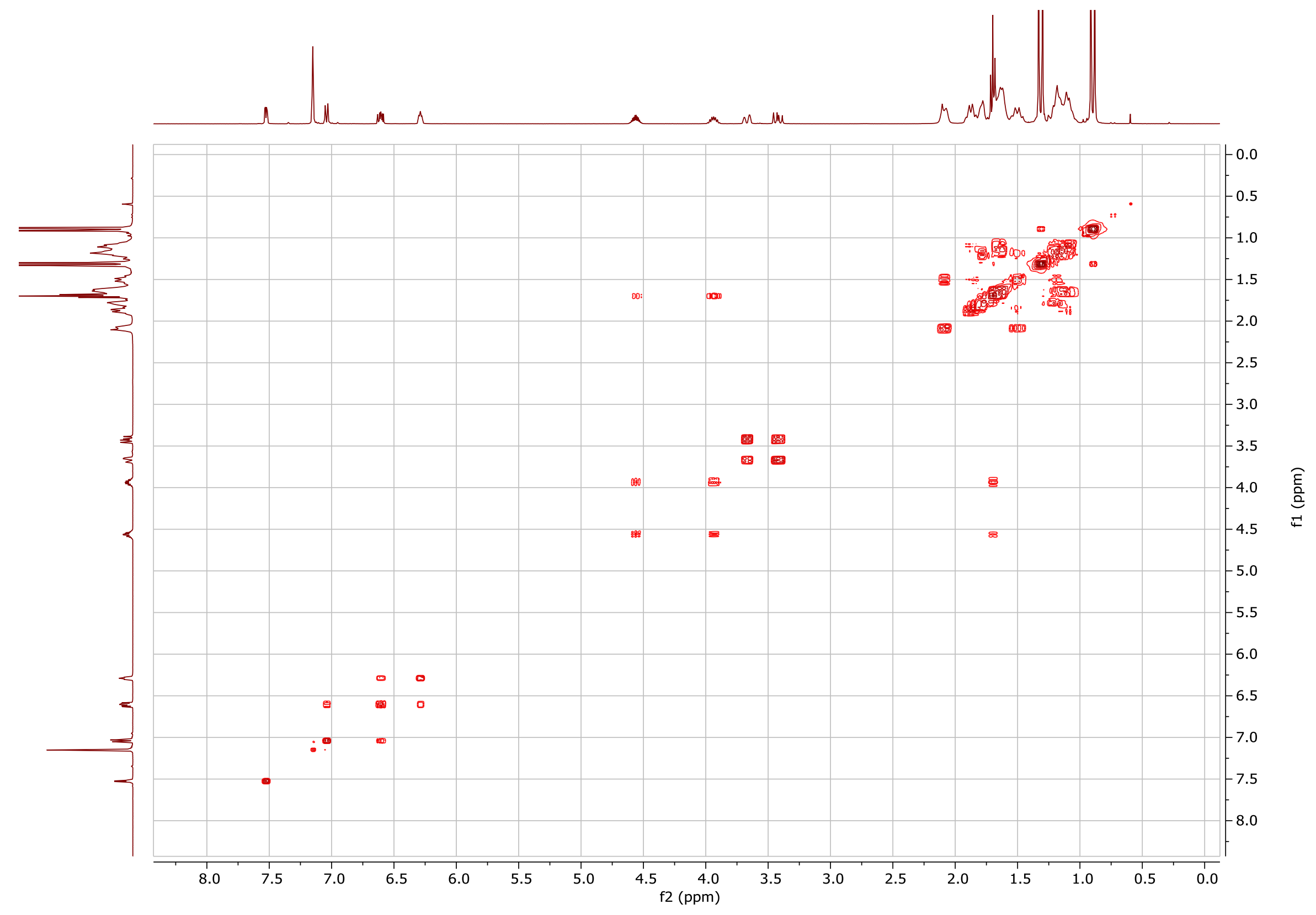

Figure S19. $\operatorname{COSY}\left(r t, C_{6} D_{6}\right)$ of RuPNN ${ }^{i m i n e}$. 


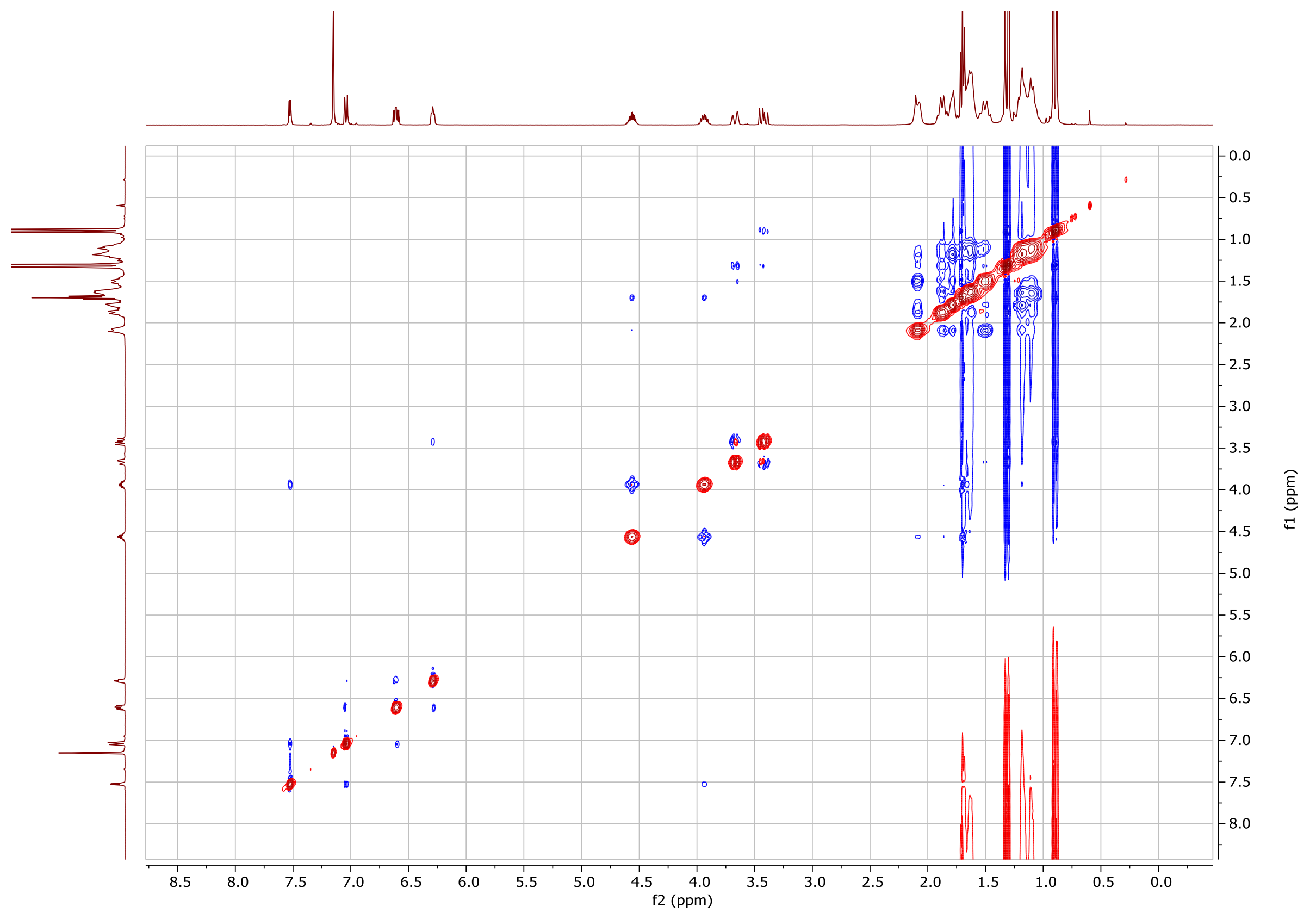

Figure S20. NOESY $\left(r t, C_{6} D_{6}\right)$ of RuPNN ${ }^{\text {imine }}$. 


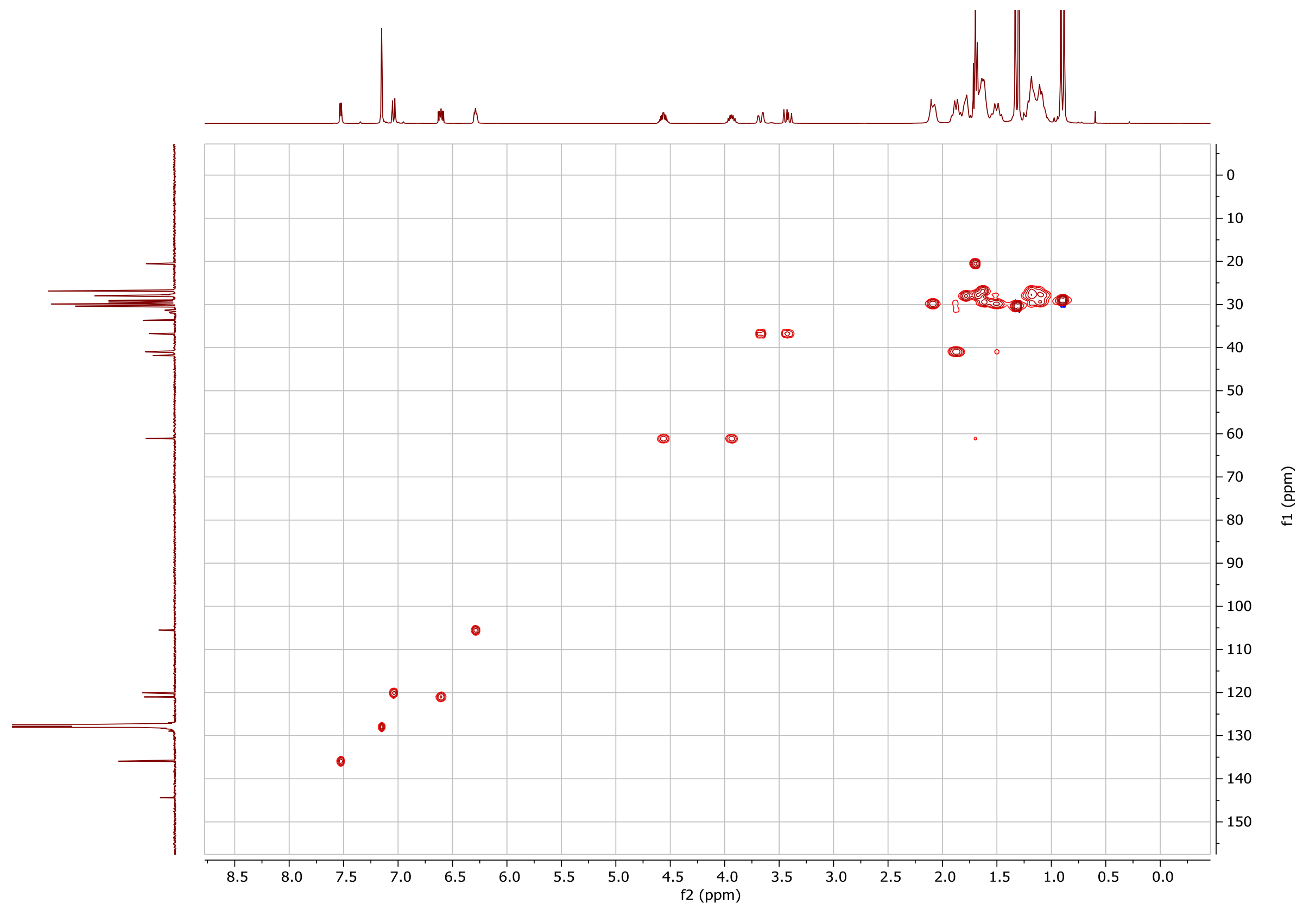

Figure S21. $\mathrm{HSQC}\left(r \mathrm{r}, \mathrm{C}_{6} \mathrm{D}_{6}\right)$ of RuPNN ${ }^{\text {imine }}$. 


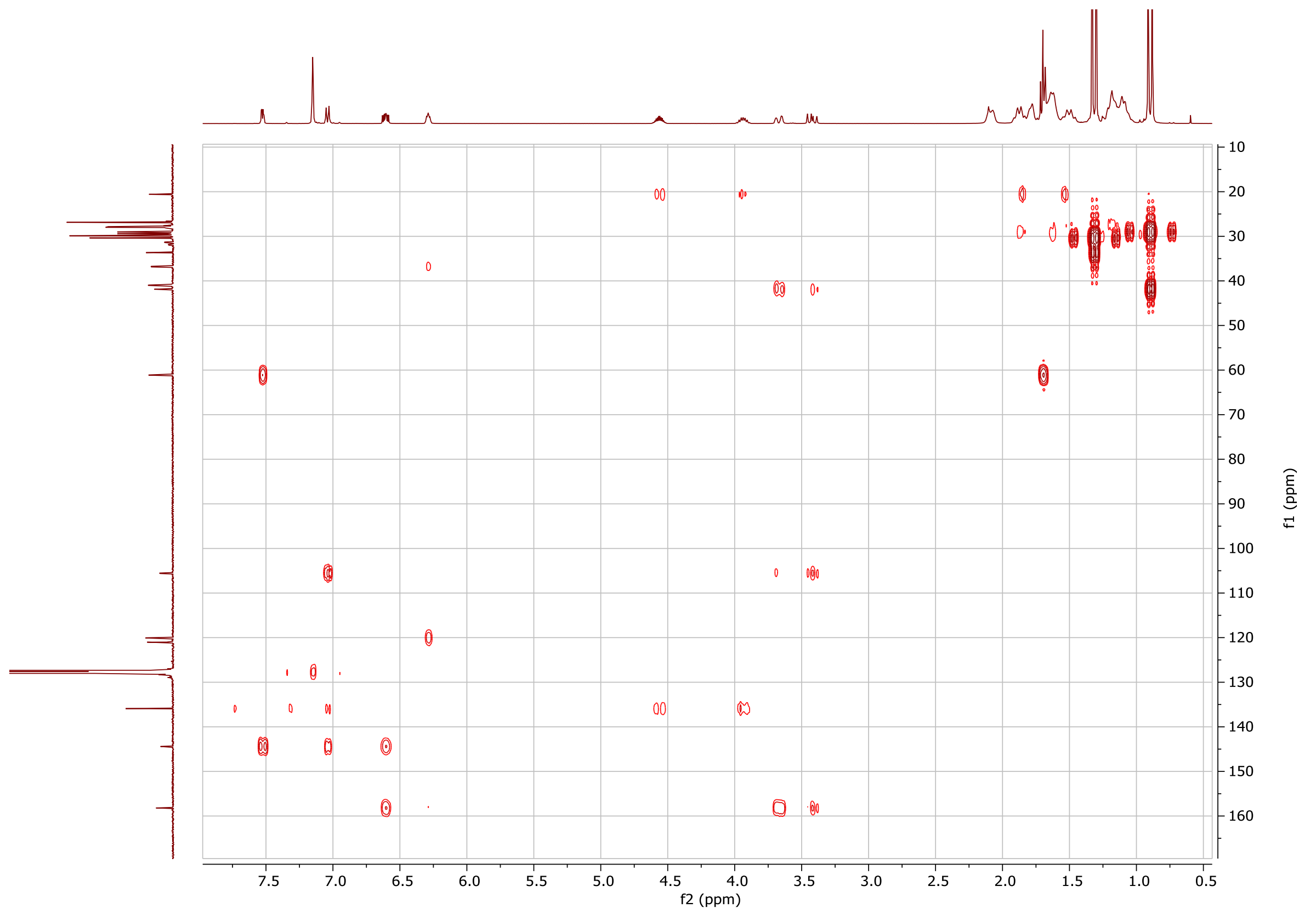

Figure S22. $\mathrm{HMBC}\left(r t, \mathrm{C}_{6} \mathrm{D}_{6}\right)$ of RuPNN ${ }^{\text {imine }}$. 


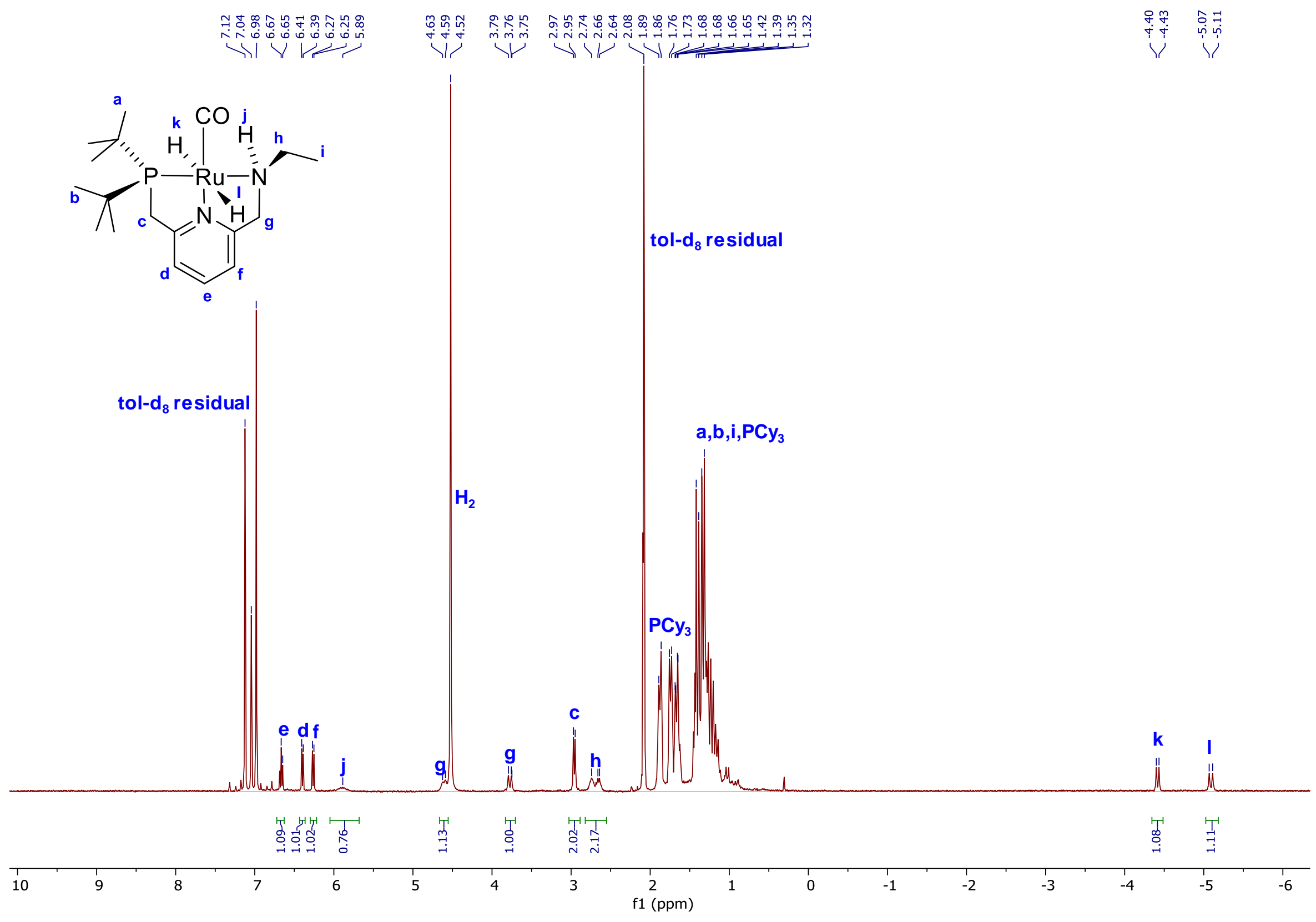

Figure S23. ${ }^{1} \mathrm{H}$ NMR $\left(-30^{\circ} \mathrm{C}, \mathrm{C}_{7} \mathrm{D}_{8}, 400.13 \mathrm{MHz}\right)$ of RuPNN ${ }^{\text {HEt }}$. 

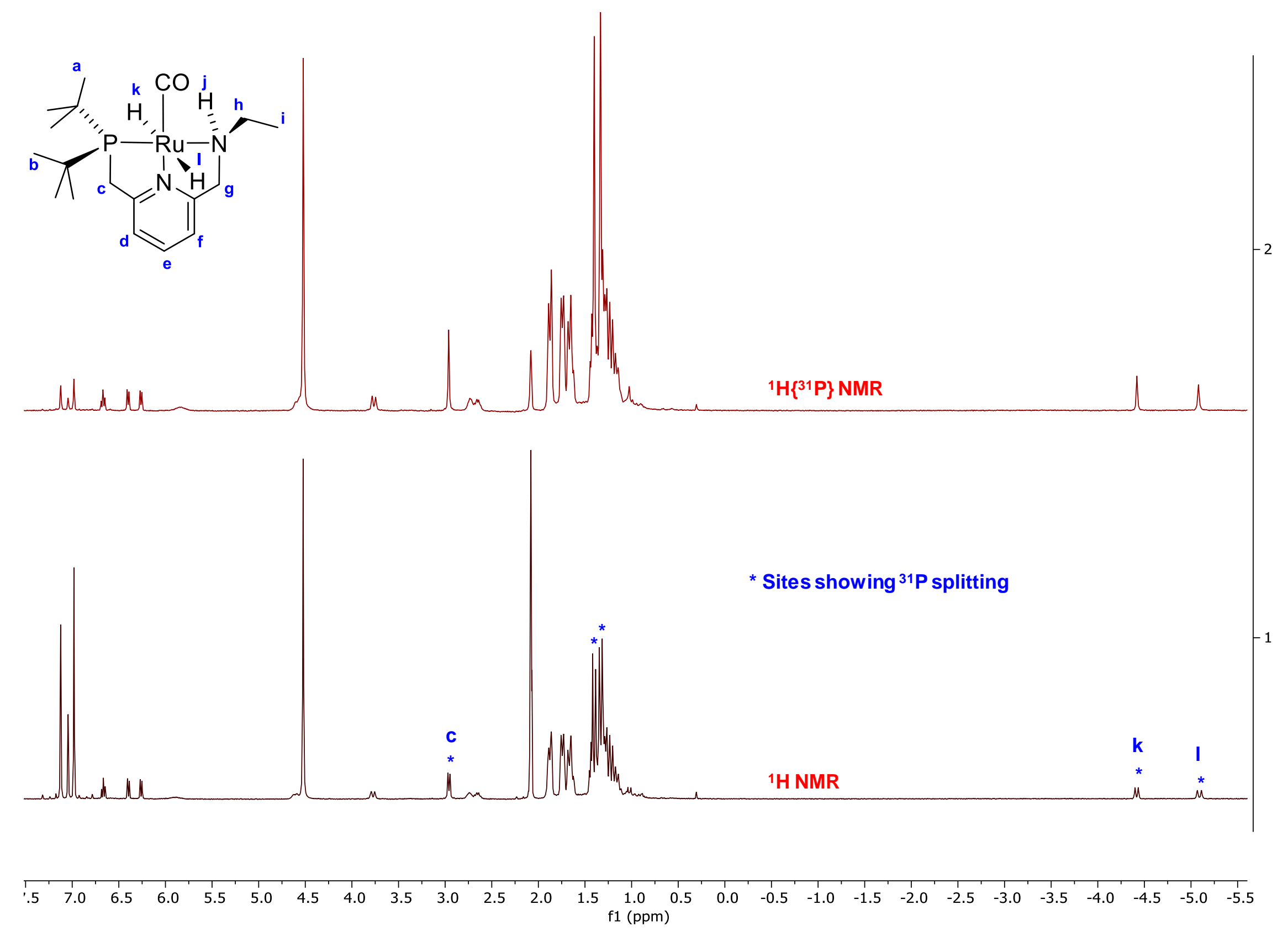

Figure S24. ${ }^{1} \mathrm{H}\left\{{ }^{31} \mathrm{P}\right\} \mathrm{NMR}\left(-30{ }^{\circ} \mathrm{C}, \mathrm{C}_{7} \mathrm{D}_{8}, 400.13 \mathrm{MHz}\right)$ of RuPNN ${ }^{\mathrm{HEt}}$ (top), compared with the undecoupled ${ }^{1} \mathrm{H}$ NMR spectrum (bottom). 

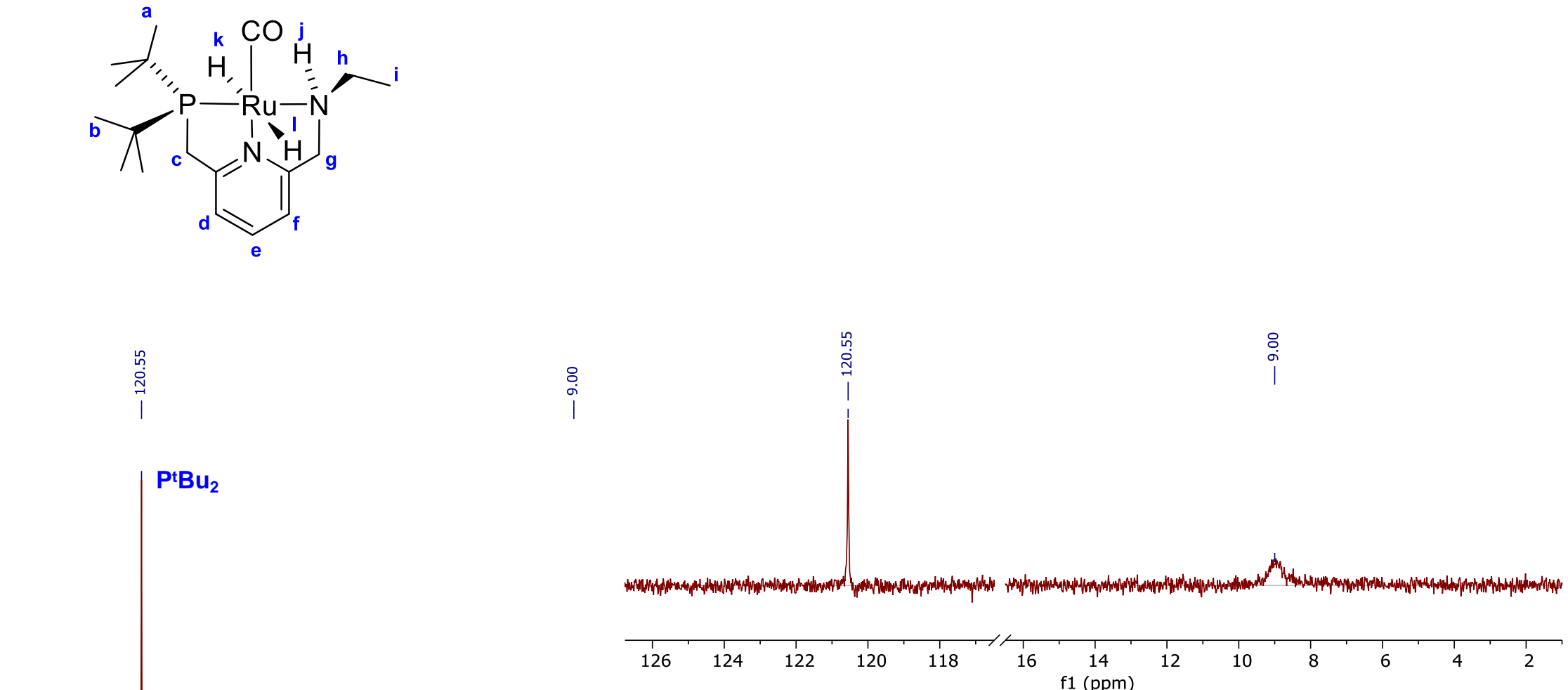

free $\mathrm{PCy}_{3}$

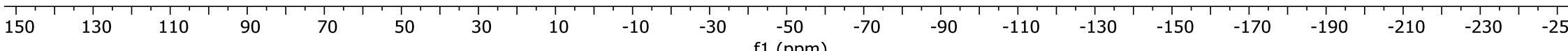

Figure S25. ${ }^{31} \mathrm{P}\left\{{ }^{1} \mathrm{H}\right\}$ NMR $\left(-30{ }^{\circ} \mathrm{C}, \mathrm{C}_{7} \mathrm{D}_{8}, 161.97 \mathrm{MHz}\right)$ of RuPNN ${ }^{\mathrm{HEt}}$. 


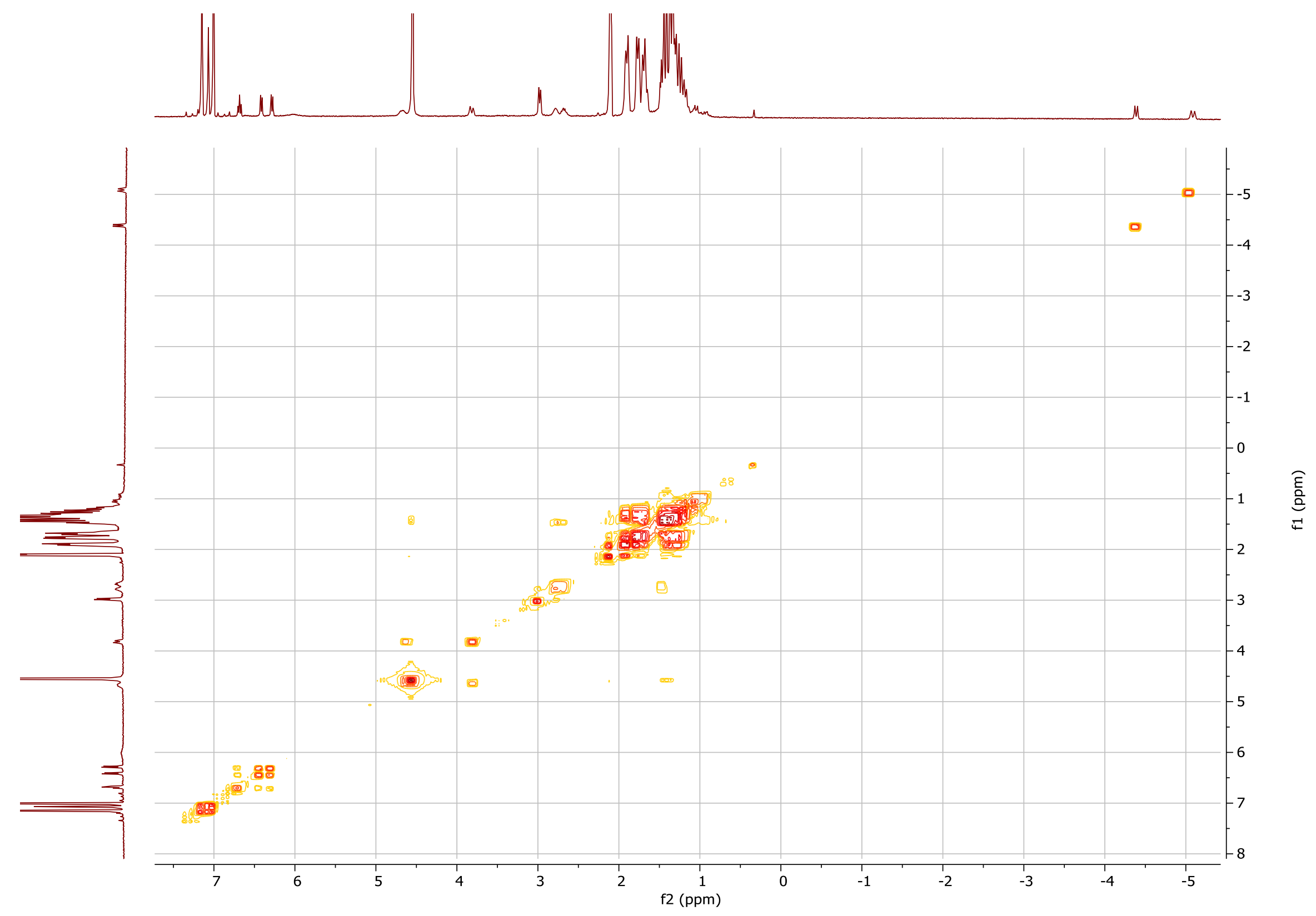

Figure S26. $\operatorname{COSY}\left(-30^{\circ} \mathrm{C}, \mathrm{C}_{7} \mathrm{D}_{8}\right)$ of RuPNN ${ }^{\mathrm{HEt}}$. 


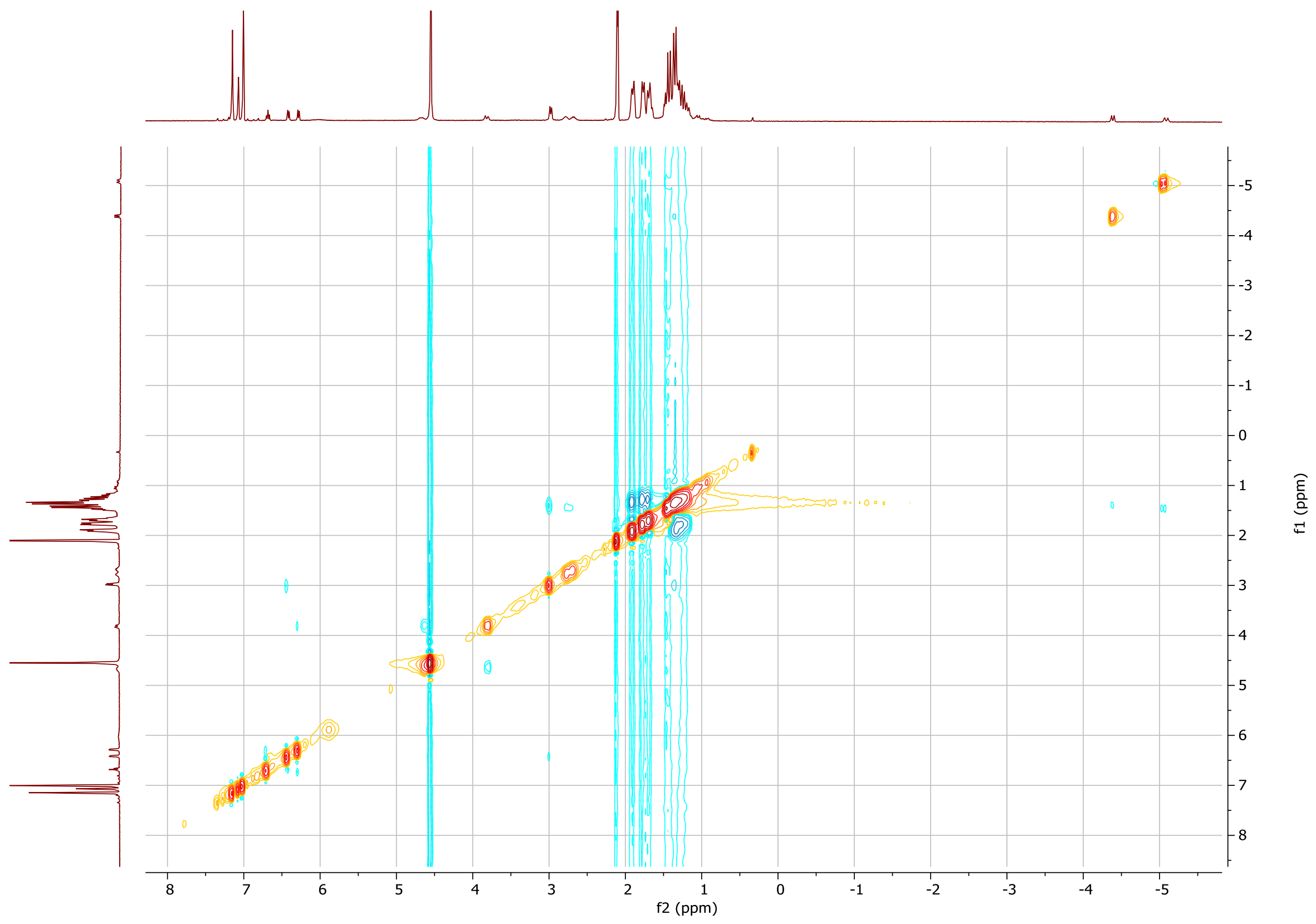

Figure S27. NOESY $\left(-30^{\circ} \mathrm{C}, \mathrm{C}_{7} \mathrm{D}_{8}\right)$ of RuPNN ${ }^{\text {HEt }}$.

S34 

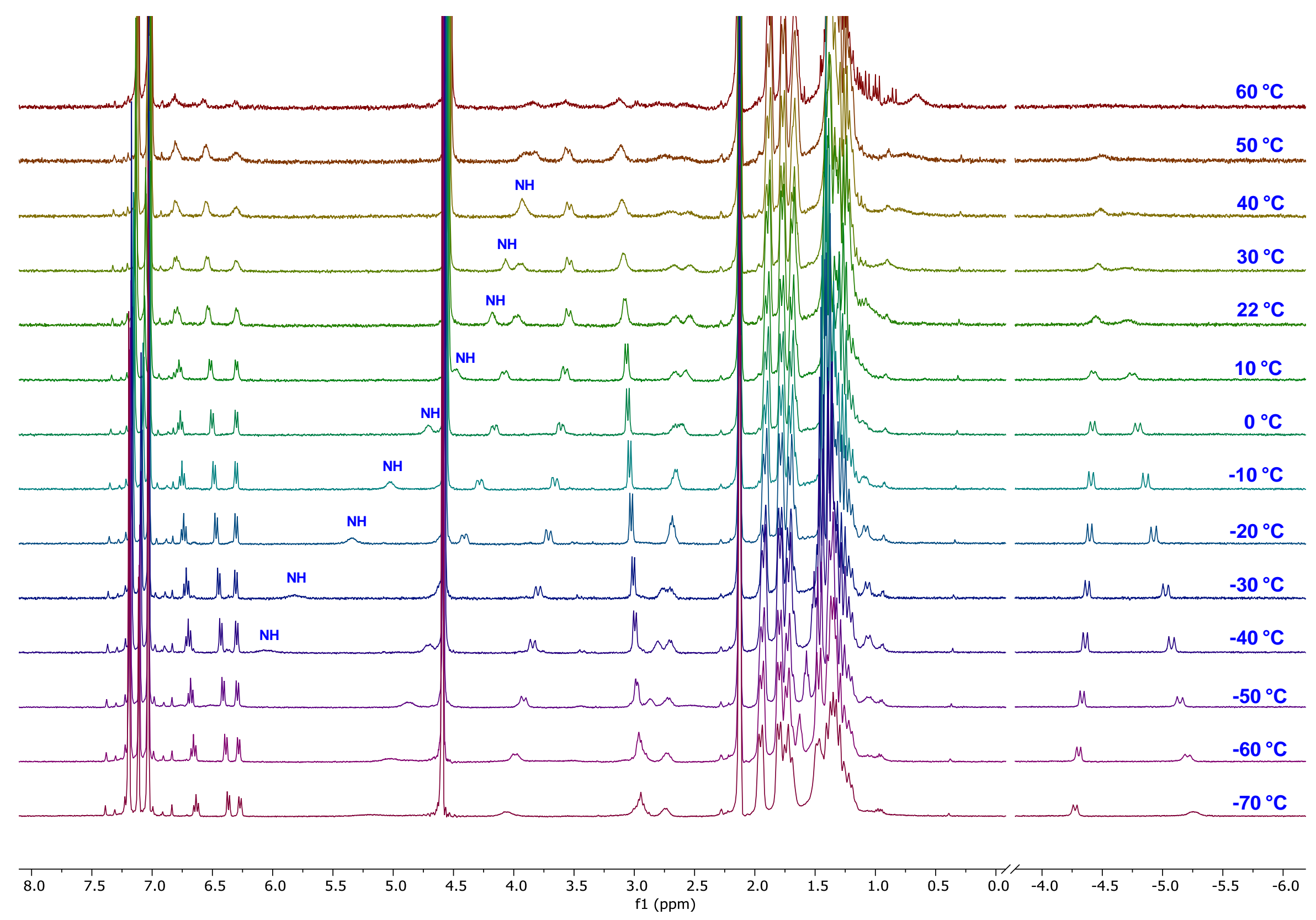

Figure S28. ${ }^{1} \mathrm{H}$ NMR $\left(\mathrm{C}_{7} \mathrm{D}_{8}, 400.13 \mathrm{MHz}\right)$ of RuPNN ${ }^{\mathrm{HEt}}$, taken from $-70{ }^{\circ} \mathrm{C}$ to $60^{\circ} \mathrm{C}$. 


\section{References}

(1) Widegren, M. B.; Clarke, M. L. Manganese Catalyzed Hydrogenation of Enantiomerically Pure Esters. Org. Lett. 2018, 20, 2654-2658.

(2) Spasyuk, D.; Vicent, C.; Gusev, D. G. Chemoselective Hydrogenation of Carbonyl Compounds and Acceptorless Dehydrogenative Coupling of Alcohols. J. Am. Chem. Soc. 2015, 137, 3743-3746.

(3) Le, L.; Liu, J.; He, T.; Kim, D.; Lindley, E. J.; Cervarich, T. N.; Malek, J. C.; Pham, J.; Buck, M. R.; Chianese, A. R. Structure-Function Relationship in Ester Hydrogenation Catalyzed by Ruthenium CNN-Pincer Complexes. Organometallics 2018, 37, 3286-3297.

(4) Oxford Diffraction (2007). Oxford Diffraction Ltd., Xcalibur Ccd System, Crysalispro Software System, Version 1.171.32.

(5) Altomare, A.; Cascarano, M.; Giacovazzo, C.; Guagliardi, A.; Burla, M. C.; Polidori, G.; Camalli, M. Sir92 - a Program for Automatic Solution of Crystal Structures by Direct Methods. J. Appl. Cryst. 1994, 27, 435-436.

(6) Betteridge, P. W.; Carruthers, J. R.; Cooper, R. I.; Prout, K.; Watkin, D. J. Crystals. J. Appl. Cryst. 2003, $36,1487$.

(7) Frisch, M. J.; Trucks, G. W.; Schlegel, H. B.; Scuseria, G. E.; Robb, M. A.; Cheeseman, J. R.; Scalmani, G.; Barone, V.; Petersson, G. A.; Nakatsuji, H.; Li, X.; Caricato, M.; Marenich, A. V.; Bloino, J.; Janesko, B. G.; Gomperts, R.; Mennucci, B.; Hratchian, H. P.; Ortiz, J. V.; Izmaylov, A. F.; Sonnenberg, J. L.; Williams; Ding, F.; Lipparini, F.; Egidi, F.; Goings, J.; Peng, B.; Petrone, A.; Henderson, T.; Ranasinghe, D.; Zakrzewski, V. G.; Gao, J.; Rega, N.; Zheng, G.; Liang, W.; Hada, M.; Ehara, M.; Toyota, K.; Fukuda, R.; Hasegawa, J.; Ishida, M.; Nakajima, T.; Honda, Y.; Kitao, O.; Nakai, H.; Vreven, T.; Throssell, K.; Montgomery Jr., J. A.; Peralta, J. E.; Ogliaro, F.; Bearpark, M. J.; Heyd, J. J.; Brothers, E. N.; Kudin, K. N.; Staroverov, V. N.; Keith, T. A.; Kobayashi, R.; Normand, J.; Raghavachari, K.; Rendell, A. P.; Burant, J. C.; Iyengar, S. S.; Tomasi, J.; Cossi, M.; Millam, J. M.; Klene, M.; Adamo, C.; Cammi, R.; Ochterski, J. W.; Martin, R. L.; Morokuma, K.; Farkas, O.; Foresman, J. B.; Fox, D. J. Gaussian 16 Rev. B.01, Wallingford, CT, 2016.

(8) (a) Lee, C.; Yang, W.; Parr, R. G. Development of the Colle-Salvetti Correlation-Energy Formula into a Functional of the Electron Density. Phys. Rev. B 1988, 37, 785-789. (b) Becke, A. D. Density-Functional Thermochemistry. III. The Role of Exact Exchange. J. Chem. Phys. 1993, 98, 5648-5652.

(9) Grimme, S.; Antony, J.; Ehrlich, S.; Krieg, H. A Consistent and Accurate Ab Initio Parametrization of Density Functional Dispersion Correction (DFT-D) for the 94 Elements H-Pu. J. Chem. Phys. 2010, 132, 154104.

(10) Hay, P. J.; Wadt, W. R. Ab Initio Effective Core Potentials for Molecular Calculations. Potentials for K to Au Including the Outermost Core Orbitals. J. Chem. Phys. 1985, 82, 299-310.

(11) Ehlers, A.; Böhme, M.; Dapprich, S.; Gobbi, A.; Höllwarth, A.; Jonas, V.; Köhler, K.; Stegmann, R.; Veldkamp, A.; Frenking, G. A Set of F-Polarization Functions for Pseudo-Potential Basis Sets of the Transition Metals Sc-Cu, Y-Ag and La-Au. Chem. Phys. Lett. 1993, 208, 111-114.

(12) Roy, L. E.; Hay, P. J.; Martin, R. L. Revised Basis Sets for the Lanl Effective Core Potentials. J. Chem. Theory Comput. 2008, 4, 1029-1031.

(13) (a) Krishnan, R.; Binkley, J. S.; Seeger, R.; Pople, J. A. Self-Consistent Molecular Orbital Methods. XX. A Basis Set for Correlated Wave Functions. J. Chem. Phys. 1980, 72, 650-654. (b) McLean, A. D.; Chandler, G. S. Contracted Gaussian Basis Sets for Molecular Calculations. I. Second Row Atoms, Z=11-18. J. Chem. Phys. 1980, 72, 5639-5648.

(14) Marenich, A. V.; Cramer, C. J.; Truhlar, D. G. Universal Solvation Model Based on Solute Electron Density and on a Continuum Model of the Solvent Defined by the Bulk Dielectric Constant and Atomic Surface Tensions. J. Phys. Chem. B 2009, 113, 6378-6396.

(15) Cramer, C. J., In Essentials of Computational Chemistry, 2nd ed.; Wiley: Chichester, UK, 2004; 378-379.

(16) (a) Zhao, Y.; Truhlar, D. G. The M06 Suite of Density Functionals for Main Group Thermochemistry, Thermochemical Kinetics, Noncovalent Interactions, Excited States, and Transition Elements: Two New Functionals and Systematic Testing of Four M06-Class Functionals and 12 Other Functionals. Theor. Chem. Acc. 2007, 120, 215-241. (b) Zhao, Y.; Truhlar, D. G. Density Functionals with Broad Applicability in Chemistry. Acc. Chem. Res. 2008, 41, 157-167. 